\title{
Naturverantwortung, Humanität und Lebensehrfurcht von Kant zu Schweitzer
}

\author{
Hans Lenk \\ https://orcid.org/0000-0003-2910-3671
}

Zwischenmenschliche ethische Verantwortung und Pflichten gegenüber Naturwesen nach Albert Schweitzers Ethik der „Ehrfurcht vor dem Leben“ und unseren „Mitgeschöpfen“. Erweiterte Verantwortlichkeit für „die Natur“ - auch für einen „humanen“ praktischen Umgang mit Ökosystemen in der drohenden Öko- und Klimakrise. „Konkrete Humanität“ in der Lebenspraxis sowie in der philosophischen Ethik - was kann dies heute bedeuten? Abschließende normative Thesen zur möglichen Verwirklichung.

Schlüsselworte: Naturverantwortung, Lebensehrfurcht, konkrete Humanität

I: Erweiterte Verantwortung „für die Natur“ und Pflichten gegenüber Naturwesen. „Humaner" Umgang - auch mit „Mitgeschöpfen “ und gar Ökosystemen

Gibt es ethische Quasirechte von oder bedingte moralische Pflichten gegenüber nicht-menschlichen Naturwesen oder „Mitgeschöpfen“)? Solche Fragen sind/waren auch in Deutschland sehr umstritten, zumindest vor dem neuen Tierschutz-Gesetz von 1998. Sie sollen hier jedoch präsentiert werden, weil die entsprechenden normativen Thesen aktuell auch die gegenwärtige tierethische

HANS LENK, professor emeritus, Karlsruhe Institute of Technology, Germany; e-mail: hans.lenk@kit.edu 
und auch allgemeine rechtliche und ethische Verantwortungsdiskussion betreffen und besonders weil sie großen Teils den einflussreichen zentralen Voraussetzungen der traditionellen (besonders auch der Kantischen) Ethik zuwiderlaufen. Sie dürften eine Revision mancher Vorstellungen der Moral- und Rechtsphilosophen veranlassen.

Das Thema ist die Diskussion und Beantwortung der Frage: Hat der Mensch ethische oder rechtliche Pflichten der Natur und den (nicht-menschlichen) Naturwesen gegenüber - und in welchem Sinne?

Am einfachsten ist es, mit Kants diesbezüglichen heute provokativ wirkenden Thesen zu beginnen.

Haben Menschen etwa nicht die Pflicht, Tiere nicht unnötig zu töten, ihnen auch möglichst nicht Schmerz und Leid zuzufügen, Tierarten zu erhalten - oder auch ein Natursystem, ein ökologisches System zu bewahren?

Ist etwa die Tierquälerei dem Menschen bloß als eine Pflicht gegen sich selbst, den Menschen, verboten, weil, wie Kant (in seiner Metaphysik der Sitten) meint $^{1}$, „dadurch das Mitgefühl an ihrem Leiden im Menschen abgestumpft und dadurch eine der Moralität im Verhältnis zu anderen Menschen sehr diensame natürliche Anlage geschwächt und nach und nach ausgetilgt wird"? Sind nur solche bloß ästhetischen (oder auf das Selbstbild und die Erziehung des Menschen selbst bezüglichen) Gründe, aber keine echt moralischen dabei relevant?

Kann man wie Kant in seiner Zeit noch folgendes meinen?

Nach der bloßen Vernunft zu urteilen, hat der Mensch sonst keine Pflicht als bloß gegen den Menschen (sich selbst oder einen anderen); denn seine Pflicht gegen irgendein Subjekt ist die moralische Nötigung durch diesen seinen Willen. Das nötigende (verpflichtende) Subjekt muss also erstlich eine Person sein, zweitens muss diese Person als Gegenstand der Erfahrung gegeben ${ }^{2}$ sein: weil der Mensch auf den Zweck ihres Willens hinwirken soll, welches nur im Verhältnisse zweier existierender Wesen zu einander geschehen kann ... Nun kennen wir aber mit aller unserer Erfahrung kein anderes Wesen, was der Verpflichtung (der aktiven oder passiven) fähig wäre, als bloß den Menschen. Also kann der Mensch sonst keine Pflicht gegen

\footnotetext{
${ }^{1}$ Immanuel Kant, Metaphysik der Sitten II, 1, $\$ 17$ [in Werke, TB-Akademie-Textausgabe, Bd. VI (Berlin: de Gruyter, 1968), 443].

${ }^{2}$ Wie kann - so stutze ich schon - eine Instanziierung eines ethischen Vernunftbegriffs als „Gegenstand der Erfahrung gegeben sein"? Ist sie nicht eher als ideales ethisches Vernunftwesen zu deuten, in jedem Falle so zu behandeln - wie Kant sogleich danach implizit bestätigt?
} 
irgendein Wesen haben als bloß gegen den Menschen, und stellt er sich gleichwohl eine solche zu haben vor, so geschieht dieses durch eine Amphibolie (Verdrehung, H.L.) der Reflexionsbegriffe, und seine vermeinte Pflicht gegen andere Wesen ist bloß Pflicht gegen sich selbst; zu welchem Missverstande er dadurch verleitet wird, dass er seine Pflicht in Ansehung anderer Wesen für Pflicht gegen diese Wesen verwechselt ${ }^{3}$.

Nach Kant hat also der Mensch gegenüber nicht-menschlichen Naturwesen keine Pflichten, sondern nur „in Ansehung “der Natur und der Naturwesen allein Pflichten „gegen sich selbst“, das heißt zur Erhaltung seiner Selbstachtung bzw. seiner Würde (z. B. der Würde der Menschheit, repräsentiert in sich selber wie in anderen) und seines moralischen Gefühls. Gibt es also gar keine positiven moralischen Rechte der Natur oder nicht-menschlicher natürlicher Wesen - nicht einmal Existenzrechte, entsprechend den auf Menschenseite zu beachtenden negativen, z.B. Vermeidungspflichten (keine mutwillige Naturzerstörung)? Hat die Natur im ethischen Sinne keinerlei „Selbstzweckcharakter“? Ist jeder Vandalismus gegenüber Naturarten und -systemen ausschließlich eine Verletzung der Pflichten des Menschen gegen sich selbst? Dürfte gar etwa ein Robinson beliebig viele Tiere quälen? Ist die Natur nur dazu da, dem ethisch „unendlich höher" gestellten Menschen als Versuchs- und Dokumentationsbereich seiner ethischen Selbstvervollkommnung zu dienen? Etwa im Sinne der oft so einseitig verstandenen, missverstandenen, christlichen Tradition des "Dominium-terrae“Gebots: „Macht Euch die Erde untertan“ (Gen.1:28)? Die Natur ausschließlich als Territorium menschlicher Willkürherrschaft?

Es existiert jedoch auch die weitere biblische Tradition des Satzes: „Und Gott, der Herr, nahm den Menschen und setzte ihn in den Garten Eden, dass er ihn bebaue und bewahre" (Gen.2:15). Die Kantische Antwort scheint idealisierend den Menschen, das Vernunftwesen, zu einem ethischen Quasigott zu machen, zum Maß aller Dinge. Vielen erscheint es heute jedoch als anthropozentrische Sophistik, einen so radikalen Bruch zwischen Natur und nicht-menschlicher Kreatur einerseits und der ethischen Verfassung des Menschen andererseits zu sehen. Kant scheint geradezu Opfer seiner strikt deontologischen absoluten Begründung der Ethik, seiner Einschränkung auf eine bewusst streng apriorisch gedeutete anthropozentrische Gesinnungsethik geworden zu sein. In der Tat

\footnotetext{
${ }^{3}$ Kant, Metaphysik der Sitten, $\$ 16,442$.
} 
scheint ein „Selbstzweck“ für eine deontologische, „reine“ sittengesetzartige Gesinnungsethik stets nur die moralische Vollkommenheit des Menschen und seines Willens aus der Vernunft des Vernunftwesens, „niemals die der außermenschlichen Natur sein“ zu können ${ }^{4}$. Oder ist die traditionelle Trennung zwischen vernunftapriorischer "reiner" deontologischer und teleologischer/konseqenzialistischer Ethik, zwischen Gesinnungs- und Konsequenzen-Ethik, zu strikt, zu absolut durchgeführt, um „vernünftig“, „wahr", zweckmäßig oder auch nur praktikabel zu sein?

In einem Vortrag über „Das moralische Recht der Natur“ meinte Spaemann 1981 sogar: Da die Natur auch ein „Selbstsein“ aufweise, insbesondere alles Lebendige "Streben“ und „Interesse" habe und insofern „stets ein Sollen“ impliziere, gebe es ein „Recht der natürlichen Wesen, so behandelt zu werden, dass wir sie in eine Güterabwägung um ihrer selbst willen einbringen“. Dies sei eine illustrierende Erläuterung des „Rechtes der Natur“. Spaemann möchte auch Kants Kategorischen Imperativ in der bekannten Formulierung: "Handle so, dass du die Menschheit sowohl in deiner Person, als in der Person eines jeden andern jederzeit zugleich als Zweck, niemals bloß als Mittel brauchst" ${ }^{5}$ abwandeln zu: „Handle so, dass nichts in der Wirklichkeit nur als Mittel, sondern es stets zugleich auch als Zweck zu gebrauchen, Maxime des Willens werde“ (kursiv v. HL). Sollte eventuell sogar Kants Grund für sein oberstes praktisches Prinzip der Sittlichkeit: (Nur) „die vernünftige Natur existiert als Zweck an sich selbst“6 auf die normal verstandene „Natur“ überhaupt ausgedehnt werden? Kann also Natur als Selbstzweck mit Rechten und als Gegenstand menschlicher Pflichten in einer entsprechend erweiterten Ethik auftreten? Kant hatte strikt zwischen „Personen“ und „Sachen "unterschieden dadurch, dass er allen Sachen „nur einen relativen Wert", eben als „Mittel“ zuschrieb, während Personen - und nur sie - durch ,ihre Natur“(!), also wesentlich, als Vernunftwesen, „absoluten Wert“ besitzen, „als Zweck an sich selbst“, d. i. als etwas, das „nicht bloß als Mittel“"gebraucht werden darf, also als Selbstzwecke, ausgezeichnet sind ${ }^{7}$. Selbst wenn man zugestehen kann, dass „der Mensch und überhaupt jedes vernünftige Wesen ... als Zweck an sich selbst,

\footnotetext{
${ }^{4}$ Dieter Birnbacher (Hg.), Ökologie und Ethik (Stuttgart: Reclam, 1980), 117.

${ }^{5}$ Immanuel Kant, Grundlegung zur Metaphysik der Sitten, in Werke, TB-Akademie-Textausgabe, Bd. IV (Berlin: de Gruyter, 1968), 429.

${ }^{6}$ Ebd.

${ }^{7}$ Ebd., 428.
} 
nicht bloß als Mittel zum beliebigen Gebrauch für diesen oder jenen Willen“ "existiert " ${ }^{8}$, also jeweils wesentlich auch als Selbstzweck gewürdigt, betrachtet und behandelt werden muss, selbst wenn er bzw. es in der Rolle eines Mittels eingesetzt wird, so kann man doch bezweifeln, dass diese Dichotomie vollständig ist. Muss jede Nichtperson zwingend eine verfügbare „Sache“von bloßem Mittelcharakter sein? Die Bestimmungen von „Mittel“ und „Zweck“ sind ohnehin relativ und können je nach Situationszusammenhang, Zielsetzung und auch nach Perspektive in gewisser Weise wechseln. Die Natur als ganze und deren Zustand kann ebenso wie der Kosmos insgesamt in der Tat nicht nur als Mittel für den Menschen oder Vernunftpersonen angesehen werden. Selbst wenn man - wie wohl auch Kant - der Meinung ist, der Kosmos als ganzer sei kein „Gegenstand“ der Handlung und der Orientierung. Obwohl jeder Mensch sich doch auch zur Welt, zum Kosmos auch als einer umfassenden, wenn auch nicht erfassbaren Ganzheit verhält: Dies zu reflektieren ist gerade eine wesentliche Aufgabe der philosophischen Anthropologie). Dürfte es nicht geradezu unzweckmäßig oder unklug sein, die Natur oder ein Ökosystem, in das auch jeweils der Mensch als ein auch biologisches Lebewesen eingebunden ist, als bloßes Mittel für menschliche Interessen zu sehen?

Das All- bzw. erdumfassende Ganze eines Systems kann nicht ausschließlich Mittelcharakter für ein Teilsystem haben, selbst wenn dieser Bezug nur in symbolischer Überfrachtung so aufgefasst werden kann oder soll. Versteht man den Menschen überhaupt auch als Naturwesen, das er trotz aller Kulturentwicklung ist und bleibt, so kann Natur an sich und jedes den Menschen einschließende Natursystem nicht bloß Mittelcharakter für ihn haben. Die Kantische absolute Einteilung und Trennung zwischen Selbstzwecken als Personen und Mitteln als Sachen müssten also differenziert werden. Man braucht nicht nur „die vernünftige Natur" als Selbstzweck anzusehen, sondern könnte auch die Ökosysteme und deren systemisch nötige Abhängigkeiten als eine Art von „Selbstzweck“ betrachten. Kant selber sieht ja in der Entwicklung und Geschichte der Menschengattung im Großen das Ergebnis eines „verborgenen Planes der Natur“ auf dem Wege zur Entwicklung einer vollkommenen Staatsverfassung sowie eines ethischen Gemeinwesens - in Kants für uns Heutige etwas missverständlicher Ausdruckweise:

${ }^{8}$ Ebd. 
„eines ethischen gemeinen Wesens" $؛$ „Der Mensch soll aus dem ethischen (=politisch-bürgerlichen, H.L.) Naturzustande herausgehen, um ein Glied eines ethischen gemeinen Wesens zu werden" ${ }^{\text {"9 }}$. Hat also nicht jedes ökologisch recht umfassende Natursystem zumindest ein relatives Existenz- und Erhaltungs-„,Recht" insofern, als zumindest jede solche Existenzrecht-Verletzung, jede Vernichtung oder Schädigung einer besonderen, außerordentlichen Begründung (etwa zur Sicherung, Erhaltung oder wesentlichen Verbesserung von Menschenleben) bedarf?

Zweifellos ist das Problem dadurch besonders akut geworden, dass der Mensch heutzutage über derartig vergrößerte Eingriffsmöglichkeiten und besonders über Zerstörungsfähigkeiten gegenüber der Natur verfügt, die alles Bisherige in den Schatten stellen. Konnte der Mensch der Antike (wie Platon schon im Kritias beiläufig feststellt ${ }^{10}$ ) eine Landschaft durch Abholzung des Waldes zerstören, konnte er schon begrenzt und lokal die Umwelt verschmutzen, so hat die Fähigkeit des modernen Menschen zur Schädigung von Natursystemen nicht nur durch eine zuvor ungeahnte Bevölkerungsexplosion, sondern besonders durch die Technisierung und Industrialisierung der Welt (vor allem in Ballungsgebieten) derart zugenommen, dass die Natur und ihre Ökosysteme nicht mehr nur lokal, sondern auch regional und kontinental oder gar global „verletzlich“geworden sind (Klima- und Umweltprobleme). Natur, Naturprozesse und Naturarten, deren Symbiosen und Ökosysteme werden gegenwärtig in extremem, zuvor nie gekanntem Maße - wenigstens und zumal negativ - äußerst nachdrücklich vom menschlichen Eingreifen bzw. von dessen Beschränkung und Lenkung abhängig. Nie zuvor hatte der Mensch sogar die Macht, alles Leben in einem Ökosystem (oder sogar regional oder kontinental bzw. gar global zu vernichten bzw. zu verstümmeln oder irreversibel zu schädigen, z. B. durch nukleare Explosionen und/oder radioaktive Kontaminierung (s. Bikini-Atoll!). Man denke an auch an die „Vermüllung“ der Ozeane durch Plastikabfälle (z.B. der landesgroße PlastikWirbel im Nordpazifik).

\footnotetext{
${ }^{9}$ Immanuel Kant, Die Religion innerhalb der Grenzen der bloßen Vernunft, in Werke, TB-Akademie-Textausgabe, Bd. VI (Berlin: de Gruyter, 1968), 96.

${ }^{10}$ Platon, Kritias, 110a-112e (Platon: Kritias. In Sämtliche Werke, Bd. 5 (Hamburg: Rowohlt 1959), 220-222].
} 
Dabei kann und wird eine von Menschen angerichtete Schädigung häufig unbewusst, unabsichtlich geschehen - als ungewollte Nebenfolge: Man denke an die vielen aussterbenden und noch mehr die gefährdeten Kleintierarten, die selbst nicht vom Menschen gejagt oder nutzbar gemacht werden, deren Ökotop jedoch verschwindet. Erlangt nicht nur die Natur als Ganzes, gewinnen nicht auch die nicht-menschlichen Kreaturen angesichts der ins Ungeheuerliche gewachsenen technologischen Macht und der dadurch entstandenen Zerstörungs- bzw. Schädigungsmöglichkeiten eine neuartige ethische Bedeutsamkeit - über alle Selbstvervollkommnungs-Ästhetik und anthropozentrische Verweisung auf Selbstachtung hinaus? In dem Maße, in dem Handlungen und Handlungsfolgen von Eingriffen des Menschen umfassende ökologische Konsequenzen nach sich ziehen können, scheinen als potentiell Betroffene auch Öko- und Natursysteme, Naturarten und Naturwesen (kurz: die Natur) ethische Relevanz anzunehmen.

Angesichts der Möglichkeit irreversibler industrieller Schädigungen aller dieser Wesen und Systeme (einschließlich des menschlichen Lebens selber) kann es dem Menschen nicht mehr nur um ihn selbst, lediglich um seine Vollkommenheit, Selbsterhaltung, Selbstbezüglichkeit gehen. Ist es für die ethische Diskussion deshalb „notwendig, die anthropozentrische Perspektive heute zu verlassen "11 bzw. wenigstens erheblich zu relativieren? Kann der Mensch aber überhaupt grundsätzlich von der anthropozentrischen Perspektive absehen? Gibt es prinzipiell eine nicht-anthropozentrische, eine überhaupt nicht „irgendwie auf den Menschen bezogene“ Ethik? Irgendwie „bezogen“ auf den Menschen ist doch jede Ethik, zumindest als auf den Adressaten aller diesbezüglich stellvertretend zu übernehmenden Verantwortlichkeiten, auch eine solche, die nicht bloß auf den Menschen als den alleinigen Verantwortungs- „Gegenstand“ bezogen ist. In dieser Hinsicht scheint Kant irgendwie doch recht $\mathrm{zu}$ haben: Pflichten und Verantwortlichkeiten können sich für die Ethik wie auch im Beachten von Rechten in der Tat immer nur auf Menschen beziehen. Der Mensch ist das einzige Wesen, das sich verpflichten und verpflichtet werden bzw. Verantwortung übernehmen kann. Dies besagt freilich nicht, dass die anthropozentrische Perspektive nicht erweitert, ergänzt werden könnte und sollte - gerade auch hinsichtlich der „Gegenstände“, auf die sich Pflichten und Verantwortlichkeiten beziehen

${ }^{11}$ Robert Spaemann, „Technischer Eingriff in die Natur als Problem der politischen Ethik“. In Dieter Birnbacher (Hg.), Ökologie und Ethik (Stuttgart: Reclam, 1980), 197. 
können. Schon dies könnte zu einer Teilrevision der Kantischen anthropozentrischen Pflichtenethik Anlass geben. Ohnehin hat die ethische Diskussion des letzten halben Jahrhunderts gezeigt, dass weder ein strikt deontologischer Ansatz noch ein strikt teleologischer oder konsequenzialistischer (insbesondere etwa rein akt-utilitaristischer) Grundentwurf absolut gesetzt oder total vertreten werden könn(t)en. Ein gemischter Ansatz, in den gewisse prinzipiell und zumindest regelhaft auf den Durchschnittsnutzen gerichtete wie auch gesinnungsethische Komponenten eingehen müssen, scheint eine „realistischere“, den moralischen Intuitionen angemessenere und zugleich praxisnähere Ethik für die fortgeschrittene Industriegesellschaft darzustellen: „Die Moral ist für den Menschen geschaffen, nicht der Mensch für die Moral“"12. Der Ausdruck, für den Menschen ' bedarf aber der genaueren Erläuterung. Ist auch als „Gegenstand“ moralischer Gebote nur der Mensch betroffen? Besser wäre es daher zu sagen: Die Moral ist für den verantwortlich handelnden Menschen geschaffen, betrifft aber rezeptiv oder passiv z.T. auch andere (Verantwortungs-),Gegenstände“.

Wie steht es dann aber mit eventuellen "Rechten“"von Natur, Naturarten/wesen und den Pflichten ${ }^{13}$ diesen gegenüber? - Aufgrund welcher Voraussetzungen und in welchem Sinne hat etwa eine Tierart ein Überlebensrecht? Gibt es Rechte von Arten, die über die der Individuen hinausgehen - möglicherweise in doppeltem Sinne? Und warum sollte die Art ein übergreifendes, u.U. höheres Überlebensrecht haben als ein Individuum? Dies sind nicht nur Fragen der rechtlichen Fundierung der Gesetzgebung und der Rechtsprechung, sondern auch der ethischen Begründung. - Man steht diesen Problemen in der anthropozentrisch bestimmten traditionellen Ethik und z.T. bis vor kurzem auch im Recht noch relativ ratlos gegenüber.

${ }^{12}$ William K. Frankena, Analytische Ethik (München: dtv, 1972), 64.

${ }^{13}$ So wurde in den U.S.A. durch ein bundesrechtliches Gesetz, das die Vernichtung einer Tierart untersagt, gerichtlich verboten, „einen Staudamm in Tennessee in Betrieb zu nehmen, weil dadurch eine bestimmte kleine Spezies, die nur an dieser Stelle existiert, vernichtet worden wäre“ (Spaemann, „Technischer Eingriff in die Natur als Problem des politischen Ethik“, 195). Kiesgruben werden z.B. auch in Deutschland aus diesem Grunde unter Naturschutz gestellt! So wird geradezu auch die „künstliche Natur"rechtlich geschützt. 
Darüber hinaus wird die ethische sowie die rechtliche ${ }^{14}$ Problematik noch gravierender: Der Mensch hat in der Bio-, Molekular- und Gentechnik die Fähigkeit erlangt, durch bio-technische Eingriffe Erbgut zu verändern, gar neue lebendige Arten durch Mutationen und genchirurgische Eingriffe zu schaffen. Eine Kröte mit dem Erbgut von sechs Elternkröten wurde schon vor dreißig Jahren wissenschaftlich „erzeugt“. Die Klonierung (erbanlagengleiche Reduplikation) von Fröschen und Mäusen ist längst durchgeführt, Kühe-Klonierung ist verbreiteter Stand der Biotechnik. Eine „Schiege“, eine „überartliche“ („trans-speziesistische“) Vermischung aus Schaf und Ziege - übrigens vermehrungsfähig - , wurde enenfalls bereits 1984 „hergestellt“(Willadsen).

Heute kann man sogar den Menschen selbst gentechnisch beeinflussen oder verändern. Und sehr umstrittene Versuche dazu gibt es bereits - für Embryo- oder pro-embryotische Zellentwicklungen. Bis zum Blastomeren-Stadium wurde sogar ein „Pro-Embryo“ von drei verschiedenen menschlichen Eltern-Genen „hergestellt“(!). Hinsichtlich seiner Pflichten und Verantwortlichkeiten stellt sich damit dem Menschen - und natürlich besonders dem naturwissenschaftlichen Forscher und (Bio- oder Gen-) Technologen/Ingenieur - eine gänzlich neuartige Problematik. Inwiefern kann der Mensch das Recht haben, künstlich neue Lebensarten zu erzeugen, Arten zu verändern - und unter Umständen seine Artgenossen oder eigenen Nachkommen selbst „eugenisch“ zu manipulieren? Menschen-Klonierung erschien bislang als absolut unethisch - wie lange noch? (Da doch Achtzellen-Blastomere und sogar längst „überzählige“ Embryonen von künstlichen Befruchtungen in manchen Ländern erlaubt und zu einem transnationalen wissenschaftlichen Embryonen-„,Handel“ geführt haben).

Eine unvoreingenommene Diskussion dieser Probleme tut not. Weder moralisch unbegründete noch rechtlich „ungestützte“ Totalverbote noch ein Absehen von jeglicher ethischen oder rechtlichen Diskussion sind hier angemessen. Sinn- und maßvolle Fortschritte scheinen allerdings nur möglich, wenn man die Selbstbezüglichkeit einer strikt(en) deontologischen Anthropozentrik

\footnotetext{
${ }^{14}$ In den U.S.A. wurde schon vor Jahrzehnten sogar das Verfahren der Herstellung für ein ölverzehrendes Bakterium patentiert (!) Lässt sich Lebenserzeugung oder ein entsprechendes Verfahren patentieren?
} 
in der Bio- und Gen-Ethik vermeidet, aber andererseits die ethischen Sonderaufgaben, -pflichten und Sonderverantwortlichkeiten des Menschen in Rechtsentwicklung und Ethik nicht vergisst.

Übrigens hatte ich schon früher betont - unabhängig und parallel zu Hans Jonas:

Neue Aktionsmöglichkeiten aktualisieren erweiterte und modifizierte Verantwortlichkeiten: z. B. gewinnt in der zunehmend technisierten Welt die Verantwortung für die Wahrung ökologischer Gleichgewichte auch für, aber nicht nur für das Interesse und Wohlergehen der Menschheit und für Natur und Kreatur ebenso an Bedeutung wie jene, die sich angesichts drohender regionaler oder globaler Überbevölkerung einstellt.

[...] mit der Ausdehnung der technisch-wissenschaftlichen Manipulations- und Einwirkungsmöglichkeiten sowie der Langzeiteffekte von unter Umständen irreversiblen Änderungen der weitgehend künstlich geprägten Umwelt entstehen weitergehende Verantwortlichkeiten, die zumindest die Relevanz moralischer Urteile (nicht nur) zeitlich, sozial und räumlich, (sondern auch essentiell) ausdehnen. In einer durch technische Eingriffe, ökonomische Abhängigkeiten, ökologische Systembedingungen näher zusammen rückenden, immer enger verflochtenen Welt kann keine Moral der bloßen Nächstenliebe genügen, sondern die Ethik muss darüber hinaus von einer zu praktizierenden Verantwortung für die gesamte Menschheit getragen werden - nicht nur für die existierende, sondern auch für die Nachwelt $^{\text {“15 }}$ (Klammer H.L.) - und für Natursysteme und Naturarten.

Insbesondere hat auch Jonas ${ }^{16}$ die Abhängigkeit der Verantwortung von der technischen Macht hervorgehoben. Er meinte, dass angesichts der ins Unermessliche gewachsenen technologischen Macht des Menschen eine sittliche Erweiterung des Verantwortungskonzeptes nötig ist: Das von der technologischen Macht des Menschen Abhängige, das Insgesamt der Betroffenen - Menschen, Natur, Tierarten eingeschlossen - sei angesichts seiner kritischen Verletzlichkeit Gegenstand der menschlichen Verantwortung in einem nie gekannten Maße geworden. "Die Natur als ein Ganzes" sei "Gegenstand menschlichen Handelns und menschlicher Verantwortung“ geworden: „ein Novum, über das ethische

\footnotetext{
${ }^{15}$ Hans Lenk, Pragmatische Vernunft (Stuttgart: Reclam, 1979), 73, $69 f$.

${ }^{16}$ Hans Jonas, Das Prinzip Verantwortung (Frankfurt/M: Insel, 1979).
} 
Theorie nachsinnen muss" (kursiv: H.L.) ${ }^{17}$. Die Irreversibilität, das Sich-aufSchaukeln von sich wechselseitig beeinflussenden Prozessen, die kumulative Addition vieler Wirkungen (synergistische Konsequenzen), umfassende Eingriffe in ökologische Systeme, kollektive Täterschaft und Verantwortung (etwa bei Großprojekten und synergistischen Effekten, die erst durch das Zusammenwirken mehrerer unterschwelliger Effekte die Schädigungsschwelle überschreiten konstituieren erweiterte Verantwortlichkeiten des Menschen und menschlicher Gemeinschaften für alle von der ins Unermessliche gewachsenen technologischen Macht Abhängigen, wenigstens für alle potentiell Betroffenen: Weil er die Macht hat, hat der Mensch Verantwortung für alles von seiner Macht Abhängige zu übernehmen.

Wie erwähnt schreibt Jonas: „Das Abhängige in seinem Eigenrecht wird zum Gebietenden, das Mächtige in seiner Ursächlichkeit zum Verpflichteten“" (Hervorh. H.L.). Und so schließe „meine Kontrolle darüber zugleich meine Verpflichtung dafür ein “18. Diese Verantwortung „als anerkannte Sorge“ um ein anderes Sein ${ }^{19}$ begründet für Jonas eine „Treuhänderschaft“ des Menschen für Natur, für Naturarten und die Biosphäre, die sozusagen als menschliches Treugut „mit eigenem moralischen Anspruch und eigenem moralischen Recht an uns aufgefasst werden müsste ${ }^{\text {“20 }}$. Er fordert also eine erweiterte, hegende Treuhänderverantwortung des Menschen für ökologische Systeme, für das Lebendige, für Natur und Leben allgemein, welche die traditionelle Verantwortung für voraussehbare Konsequenzen meines eigenen Handelns (und die entsprechende Handlungsresultats-Verantwortung bzw. -Schuld ${ }^{21}$ ) weit übersteigt. Insbesondere meint Jonas, prinzipiell sei „alles Lebendige“ „in seiner Bedürftigkeit und Bedrohtheit“ möglicher und realer „Gegenstand von Verantwortung“. Nur zum Menschen freilich gehöre es - ähnlich wie bei Kant und wie erwähnt - als eine, seine spezifische moralische „Auszeichnung“, dass er - und nur er allein - Verantwortung tragen

\footnotetext{
${ }^{17}$ Ebd., 27.

${ }^{18}$ Ebd., 174 ff.

${ }^{19}$ Ebd., 392.

${ }^{20}$ Ebd., 28 ff.

${ }^{21}$ Fälschlich meinte Jonas zunächst, das neue erweiterte Macht-Abhängigkeitsmodell ex ante der Verantwortlichkeit für das „Zu Tuende“ löse das herkömmliche post hoc - Modell der Schuld-Verantwortung ab, hat das aber nach unseren Diskussionen in Deutschland und Nordnorwegen korrigiert.
} 
kann - für seinesgleichen, aber auch für anderes Lebendiges und auch für „die Natur" als Verantwortungsgegenstand“22.

Die Ethik einer erweiterten Verantwortlichkeit auch i. S. von Jonas bezieht sich in den Pflichten also weiterhin auf den Menschen als handelndes Subjekt sowie auf menschliche Gemeinschaften, aber sie weicht von Kants „Metaphysik der Sitten“ und deren ethischer „Tugendlehre“ insofern ab, als sie Pflichten auch gegenüber nicht-menschlichen Verantwortungs-„,Gegenständen“ annimmt. Die traditionelle ganz symmetrische $\mathrm{Zu}$ (einander)ordnung von ethischen Pflichten und moralischen Rechten wird hier aufgegeben oder geändert - zugunsten einer Asymmetrie der Verantwortung und der zugeordneten Rechte und Pflichten, die der Macht- und Kontrollasymmetrie entspricht. „Mit der Machtergreifung der Technologie ${ }^{“ 23}$ habe sich, meint Jonas, die Verantwortung des Menschen und der Menschen wesentlich erweitert - bis hin zu einer Verantwortung für den Zustand der Natur, ,den Zustand der Biosphäre und des künftigen Lebens der Menschenart“ sowie für die „Zukunft der Natur“ und der Arten in ihr ${ }^{24}$. Nicht mehr nur die Menschheit als Ganzes, einzelne Menschen als Handlungspartner und die Zukunft der Menschheit sowie von unseren Handlungen abhängige Menschen sind Gegenstand der Verantwortung, sondern der Bereich des Verantwortbaren, des der Verantwortung Unterstehenden hat sich im entsprechenden Verhältnis mit der Ausdehnung technologischer Macht auf die gefährdete Natur, Tierarten usw. erweitert.

Systematisch-ethisch bedeutet dies eine Teilverabschiedung oder wenigstens wesentliche Erweiterung der traditionellen aufklärerischen Vernunftethik, die Selbstzwecke nur in Vernunftwesen sah: Jedes Lebendige, ja, Natursysteme an sich oder die „Natur“ müssten in diesem Sinne ${ }^{25}$ in einer erweiterten Verantwortungsethik als Selbstzwecke anerkannt werden - wie bereits bei Albert Schweitzer (s.u.). Ein solcher Selbstzweck konstituiert gleichsam ein moralisches Quasirecht auf Bestehen, Weiterexistenz, Erhaltung und Förderung. Diese inhaltliche moralische Änderung muss beachtet werden. - Oder es wird die methodologische Variante gewählt: Die Kantische Symmetrie zwischen Rechten und Pflichten wird

\footnotetext{
${ }^{22}$ Jonas, Das Prinzip Verantwortung, 185, 27.

${ }^{23}$ Ebd., 229.

${ }^{24}$ Ebd., 245 ff.

${ }^{25}$ Außer wohl Krankheitserreger wie das Pockenvirus usw.
} 
dann aufgehoben (s.u.): Faktisch jedoch kann beides kombiniert werden das "Oder" in der Hauptthese ist einschließend gemeint.

Zwar ist es nach wie vor eine wesentliche Auszeichnung des Menschen, dass nur er Verantwortung und Pflichten haben kann - aber eben nicht mehr nursich selbst oder seinesgleichen gegenüber, sondern eben auch anderen Selbstzwecken, schließlich gar der Natur gegenüber und hinsichtlich ihrer (Öko-)Systeme und deren Symbiosen. Die traditionelle ethische "Selbstvergötzung" des Menschen (nur er ist Gegenstand ethischer Verantwortung und Verpflichtung) ist damit aufgegeben, ohne dass freilich seine Auszeichnung als ethisches Subjekt (als Träger der Verantwortung und Verpflichtung) aufgegeben wäre.

Schon traditionell hatte allerdings im Juristischen die Zuschreibung von Rechten zu nicht-menschlichen Naturwesen ihre Schwierigkeit. Vordergründig zog man sich auf den Standpunkt zurück, wo kein Anwalt und kein Ankläger sowie kein rechtlicher Anspruchsträger existieren, könnten auch keine Rechte sein bzw. zuerkannt werden ${ }^{26}$.

Bei einer Versammlung der Gesellschaft für Rechtspolitik unter Anwesenheit fast aller Präsidenten der obersten deutschen Gerichte und sogar des Bundespräsidenten wurde das Argument in die Diskussion gebracht, ungeborene einzelne und kommende Generationen hätten keine Rechtsansprüche geltend zu machen, da sie "noch nicht einmal existierten “27. Insbesondere sei ein allgemeiner Rechtsanspruch eines noch nicht existierenden Wesens juristisch nicht kodifizierbar. Etwas Nichtexistierendes könne kein Rechtsträger sein, besonders nicht Inhaber eines Rechtes auf Existenz.

Gibt es aber nicht die (z. B. von Birnbacher vorgeschlagene) Möglichkeit, bedingte Rechte auf Wohlergehen, Lebensqualität usw. zu formulieren, nur für den Fall, dass diese künftigen Wesen oder Generationen existieren?

Solche Quasirechte könnten sich in der Tat auch auf die noch nicht geborenen menschlichen Generationen beziehen, ohne dass potentielle einzelne (noch

${ }^{26}$ „Die Annahme, dass irgendetwas außer dem Menschen 'Rechte` besitzen könnte, ist gänzlich unhaltbar“, meinte der Passmore [John Passmore, „Den Unrat beseitigen“, in Dieter Birnbacher (Hg.), Ökologie und Ethik (Stuttgart: Reclam, 1980), 229]: Sonst müsste ja auch „das Land, das ein schlechter Farmer in den Fluss abrutschen lässt", das Recht gehabt haben, „an Ort und Stelle zu bleiben“. Es hatte aber „keinerlei Recht“ dazu.

${ }^{27}$ Vgl. Hans Lenk, „Herausforderung der Ethik durch technologische Macht“, Bitburger Gespräche 1981, Gesellschaft für Rechtspolitik Trier (München: Beck): 5-38. 
nicht gezeugte) Nachkommen nunmehr ein „subjektives“ „Recht“ darauf hätten, geboren zu werden. Als Träger von Einzelrechten müssten sie ja schon existieren, um überhaupt ein Recht im engeren Sinne zu besitzen geboren zu werden ${ }^{28}$. Es „mögen die Nachgeborenen zwar ein Recht darauf haben, eine halbwegs unversehrte Umwelt hinterlassen zu bekommen, niemals aber ein Recht darauf, allererst zu existieren" ${ }^{\text {229. }}$.

Mich veranlasste all das damals (1982) zu dem Vorschlag, einen Ombudsmann oder einen Bundesbeauftragten für die Interessen und Rechte künftiger Generationen einzuführen ${ }^{30}$. Ebenso könnte man einen Beauftragten für nichtmenschliche Naturkreaturen ernennen, der deren moralische und rechtlich relevante „Quasirechte ${ }^{\text {“31 }}$ auf Existenz und Erhaltung usw. vertreten könnte ${ }^{32}-$ sozusagen einen parlamentarischen Naturschutzbeauftragten. (Ein Umweltministerium gibt es ja immerhin in Deutschland, wenn auch leider kein umfassendes „Umweltgesetzbuch“33).

Man könnte also sozusagen ein "Quasireflexrecht" von natürlichen Arten und Natursystemen für die ethische und auch juristische Verantwortlichkeit des/der Menschen anerkennen. Das ist die normativ-inhaltliche Teilthese der disjunktiven Hauptthese. Darüber hinaus könnten projizierbare Quasi-Ansprüche

${ }^{28}$ In den U.S.A. hat jedoch ein schwerstbehindertes Kind (per Rechtsanwalt natürlich) seine Eltern verklagt, indem ein Recht „auf Nicht-geboren-Werden“ in Anspruch genommen wurde, so las ich vor einigen Jahren.

${ }^{29}$ Birnbacher, Ökologie und Ethik, 125.

${ }^{30}$ Im Übrigen könnte man, um Missverständnisse und Interpretationsschwierigkeiten zu vermeiden, in der Tat nur von moralischen und (evtl. auch juristisch zu kodifizierenden) projizierten Quasirechten natürlicher Arten und natürlicher Systeme sprechen, ohne ihnen einklagbar direkte AnspruchsRechte im engeren juristischen Sinne zuzugestehen. Die Quasirechte wären jeweils von Menschen ethisch stellvertretend zu artikulieren und zu vertreten. Rechtlich könnte ein Beauftragter wie ein Ombudsmann usw. diese Quasirechte stellvertretend wahrnehmen.

${ }^{31}$ Jedenfalls ist die Begründung von Rechten ausschließlich auf die Vertretung subjektiver Ansprüche weder rechtsphilosophisch noch ethisch vertretbar, zumal manche Rechtskodizes schon sogenannte Reflexrechte in sog. „Programmsätzen“ enthalten, die Begünstigungen oder Gewährleistungen garantieren, aber - wie zum Beispiel das vieldiskutierte „Recht auf Arbeit“ - keine adressierbaren Ansprüche geltend zu machen erlauben.

${ }^{32}$ Auch bei Kleinkindern und Schwachsinnigen nimmt ja ein Vormund die Rechtsvertretung wahr.

${ }^{33}$ Diesbezügliche Versuche seit 2006 „scheiterten“ 2009. Sie gingen aber z.T. in rechtliche Regelungen von Naturschutz, Landschaftspflege, Wasserhaushalt, Stoffe-Transporte usw. ein. 
nicht-menschlicher Verantwortungsgegenstände rechtlich durch gesetzlich Beauftragte vertreten werden. Tierschutzbeauftragte gibt es ja zum Teil schon und die Tierschutzgesetzgebung hat die anthropozentrische Deutung der juristisch kodifizierbaren und verfahrensmäßig vertretbaren subjektiven Anspruchsbegründung der Tiere als bloßer „Sachen“ auch schon zum Teil überwunden.

Natürlich könnte es gesetzgeberische und juristische Schwierigkeiten bei der justitiablen Kodifizierung der Quasirechte und der entsprechenden Verantwortlichkeit geben. Doch wie in Fällen der „unterlassenen Hilfeleistung“ Einzelnen gegenüber oder des Verursacherprinzips im Umweltschutz könnten auch hier Wege gefunden werden. Ähnlich wie bei den Reflexrechten könnten wohl moralische Quasirechte der genannten Art explizit in manche Gesetzkodizes (wie bisher wohl schon in Umweltrechte und das Tierschutzgesetz von 1998) eingehen.

Diese neue deutsche Tierschutzgesetz korrigierte wenigstens den traditionellen juristischen Status der Tiere als „Sachen“34, bezeichnet diese nunmehr als „Mitgeschöpfe“, deren „Leben und Wohlbefinden zu schützen“ ist: „Niemand darf einem Tier ohne vernünftigem Grund ${ }^{35}$ Schmerzen, Leiden oder Schäden zufügen“ $(\$ 1)$. $\$ 2$ regelt ,angemessene“ Ernährung, Pflege, Unterbringung sowie "verhaltensgerechte“ Behandlung und „artgemäße“ Bewegungsmöglichkeit, $\$ 3$ Tiertransporte, $\$ 4$ die „Tötung von Tieren“ („nur unter Betäubung“, „nicht mehr als unvermeidbare Schmerzen“(?)), $\$ \$ 7-9$ Tierversuche (Beschränkung auf Krankheitserkenntnisse, -vorbeugung, -behandlungs-(möglichkeiten), Stoffeund Produktprüfungen, Umweltgefährdung sowie Genehmigungen und Expertenpflichtigkeit von (Tier-)Impfungen, Blutuntersuchungen usw. (Die Einzelparagrafen außer recht summarisch $₫ 2$ behandeln allerdings nur die recht detaillierten Regelungen der Tötungsmodalitäten, erwähnen aber gar nicht mehr das „Wohlbefinden“).

Michael Bartsch, der Rechtsanwalt, mit dem ich um 1980 Seminare über Kants Rechtsphilosophie durchführte, meinte schon damals - also lange vor dem Tierschutzgesetz -, dass auch juristisch in der rechtstechnischen Praxis (wenn auch nicht in der Rechtstheorie) die Einführung des rechtlichen Schutzes nichtmenschlicher Wesen kein Problem sei:

\footnotetext{
${ }^{34}$ Zum neuen Stand der Rechtsordnungsdiskussion dieses Themas s. a. Jörg Luy, „Welche Rechte haben Tiere?", Spektrum der Wiss., Dez. 2016: 80-84.

${ }^{35}$ Das ist zweifellos ein recht vager Gummiparagraph geblieben!
} 
Rechtstechnisch ist der Schutz der Natur kein Problem. In der Tierschutzgesetzgebung und - recht neu - bei der internationalen Regelung des Artenschutzes ist der Gedanke positiv rechtlich schon vollzogen. Wenn ich richtig sehe, werden stets Unterlassungspflichten statuiert; sie können an der unerwünschten Handlung selbst (z. B. Quälen des Tieres) anknüpfen oder auch an ganz entlegenen Spätfolgen (z. B. das Importieren von Elfenbein; damit wird das Abschlachten von Elefanten in wirtschaftlichem Reflex verhindert (oder wenigstens vermindert, H.L.), weil das gewonnene Elfenbein nur im Export absetzbar ist). Die Überwachung wird Behörden (Zoll, Amt für Gewässerschutz, Staatsanwaltschaft) übertragen, die kraft Amtes auf Einhaltung der Unterlassungspflichten dringt und die Einhaltung der Regeln durch Strafen und Auflagen erzwingt.

Wer oder was bei diesen Unterlassungspflichten eigentlich der Träger des Rechtes ist, ist rechtstechnisch (aber nicht philosophisch, H.L.) ohne Bedeutung. In ähnlicher Weise könnte man Kunstwerke unabhängig von der Eigentums- und Besitzzuordnung vor der Vernichtung schützen. Das ethisch als plausibel erkannte Ziel (z. B. Naturschutz) kann also rechtstechnisch auch ohne systematische rechtstheoretische Herleitung in die Praxis umgesetzt werden.

Wie weit sich der Schutz erstrecken soll, ist nicht nur im Einzelfall, sondern auch in der Theorie mit problematischen Abgrenzungsfragen beschwert. Dass generell nur Unterlassungspflichten und nicht Handlungspflichten rechtlich statuiert werden, erscheint mir sachgerecht. Einen Schutz der Natur vor sich selbst könnte man statuieren, z. B. also die Pflicht schaffen, ein Mittel gegen den Schädling, der ... (damals fast, H.L.) alle Ulmen vernichtet(e), zu entwickeln und anzuwenden. Aber die Theorie stößt an die Grenze, die ihre Herleitung aus dem Gefühl der Selbstbedrohung erkennbar macht. Hätte man beispielsweise das Aussterben der Saurier verhindern müssen? Ich meine nicht. Eine Hege der Natur, also eine Bewahrung des Status quo scheint mir zu weit zu gehen. Es sollte nicht ein Handeln, sondern nur ein Unterlassen geboten werden mit der Ausnahme von Fällen, in denen ein früheres Handeln erst das Risiko (z. B. das Risiko des Aussterbens einer Tierart) geschaffen hat ${ }^{36}$.

Wären im Sinne dieser übergreifenden Reflexrechte Arten gegenüber Individuen rechtlich ausgezeichnet? Es scheint so, dass eine ethische und rechtliche Verpflichtung des Menschen besteht, seine Art und andere Arten nicht aussterben zu lassen, ohne dass nicht schon gezeugte Einzelwesen irgendwie ein Einzelrecht

\footnotetext{
${ }^{36}$ Michael Bartsch, brieflich an den Autor ca. 1981.
} 
auf Geburt hätten. Natürlich ergeben sich bei Menschen als Rechtssubjekten durchaus noch andere Probleme als bei nicht-menschlichen Trägern von Quasirechten.

Birnbacher hebt darüber hinaus hervor, dass wir ,im Falle von (leidensfähigen, HL) Tieren etwas sagen können, was wir im Falle von Landschaften und Flüssen nicht sagen können, nämlich, dass wir ihnen gegenüber die Pflicht haben, sie nicht zu quälen, dass wir es ihnen schuldig sind, sie nicht unnötigen Schmerzen auszusetzen “37. Im Gegensatz zu den Landschaften (denen gegenüber nicht, sondern nur ,in Ansehung“ derer wir Pflichten übernehmen - etwa gegenüber potentiellen künftigen Betrachtern, s.o.) haben Tiere den Charakter von QuasiRechtssubjekten als leidensfähigen Kreaturen, da „wir Pflichten und damit Verantwortung gegenüber der außer-menschlichen Natur insoweit haben, als diese leidensfähig ist" - im Sinne der heute sog. pathozentrischen Bioethik seit J. Bentham $^{38}$.

Birnbacher möchte also bei der symmetrischen Zuordnung bleiben: „X hat immer dann ein Recht gegenüber Y, wenn Y eine Pflicht gegenüber X hat ${ }^{\text {“39 }}$ (Das gilt für ihn wohl sowohl moralisch als auch rechtlich.) - „Die Existenz eines Korrelats“, des Gegenstandes der menschlichen Verpflichtung, ist für ihn also stets erforderlich. So hätten Tiere als existierende Rechtsträger entsprechend moralisch „ein Recht“, „,vor Schmerzen bewahrt zu werden“, soweit sie „fühlende und leidensfähige Lebewesen “ sind. Aber bei Unterstellung der vollständigen Symmetrie müssten auch Tieren moralische und eventuell rechtliche Pflichten zugeordnet werden (TierschutzG). (Klingt es nicht absurd, aus früheren Jahrhunderten Berichte von sog. „Hinrichtungen“ von Tieren und entsprechenden Gerichtsverfahren gegen diese zu hören, wenn sie Menschen getötet hatten und nun mit ihrem Leben dafür sühnen mussten?)

Die Auszeichnung der Leidensfähigkeit als Kriterium moralischer Relevanz lässt sich zwar aus einer utilitaristischen Moralbegründung (nach Bentham [1789], der dieses Kriterium auch als erster formuliert hatte) ableiten, scheint aber ähnlichen Schwierigkeiten gegenüber zu stehen wie die Auszeichnung aufgrund der Kriterien Vernunft, Bewusstsein, Interesse (J. Feinberg) usw.: Warum sollte

\footnotetext{
${ }^{37}$ Birnbacher, Ökologie und Ethik, 124.

${ }^{38}$ Ebd., 121.

${ }^{39}$ Ebd., 125.
} 
eine Vernichtung, Schädigung von nicht mit Gefühl, Empfindung oder Nervensystemen, Interesse ausgestatteten kleineren Lebewesen moralisch irrelevant sein? Fängt das moralische „Recht auf Leben“ erst mit der Leidensfähigkeit oder mit dem „höheren“Interesse an? Die Abgrenzung des Gegenstandes der Verantwortung schiene hier willkürlich, zumal es zweifellos Grenzfälle der Bemerkbarkeit oder Darstellung von „Leiden" oder entsprechend „Interesse“ gibt (vgl. auch unten).

Allerdings: weder das Leidenskriterium noch das Interessekriterium Feinbergs reichen hier, um die ethischen Intuitionen angemessen zu erfassen. Eher scheint es hier sinnvoll zu sein, die wechselseitige Symmetrie von Pflichten und Rechten bzw. Quasirechten aufzugeben, (nämlich den Satz: „Nur wo Rechte existieren, gibt es Pflichten - und umgekehrt") und - nunmehr entgegen der Kantischen Auffassung - Pflichten auch gegenüber nicht-menschlichen Wesen der Natur und für die Behandlung von Ökosystemen und natürlichen sowie sogar gemischten zugleich „naturalen“ und menschen-gemachten oder -geprägten Gesamtsystemen einzuführen. Dementsprechend ließen sich - vielleicht in geeigneter gradweiser Abstufung - moralische Quasirechte „konstruieren“, eventuell später einmal sogar juristisch ${ }^{40}$ einführen, kodifizieren und etwa durch sog. Ombudsleute vertreten.

Um noch einmal Bartsch zu zitieren:

Was rechtstechnisch durch den Beschluss des Gesetzgebers leicht zu schaffen ist (nämlich Pflichten ohne korrelierende Rechte), bereitet in der Theorie (jedoch) Schwierigkeiten, die aus der kantischen Entscheidung herrühren, nur vernunftbegabte Wesen als Selbstzwecke anzusehen". - Dann müsste eben die Kantische Anthropozentrik der ethischen Gegenstände abgeändert werden: „Nicht erst der heute in den Vordergrund getretene Gedanke des Bestandsschutzes auch für die Natur erzwingt eine Modifikation der kantischen Theorie, sondern schon ihr eigenes

${ }^{40}$ So wie man bei den oben genannten Reflexrechten, die allerdings meist z. B. auch mittelbare Gewährleistungsrechte umfassen, welche nicht Abwehrrechte sind, die strikte umkehrbar-eindeutige Zuordnung von einklagbaren Rechten und eindeutig zuzuordnenden Pflichten aufgegeben hat, könnten auch Pflichten existieren, denen keine vollentwickelten Rechte - insbesondere nicht die Rechte einzelner selbstvertretungsberechtigter Wesen - gegenüber stehen. Dies würde sowohl beim Problem der nichtmenschlichen Naturarten und -systeme als auch bei anderen ethischen und rechtlichen Gegenständen der Verantwortung eine vermittelnde Lösung ermöglichen (wie auch beim o.a. Problem der noch nicht existierenden, nicht gezeugten Nachkommen). 
haarspalterisches Ergebnis, z. B. Tierquälerei nur ,in Ansehung des Menschen`abzulehnen“: Wenn Kant die Tierquälerei nur, in Ansehung des Menschen' missbilligt, dann spricht er dem Tier nicht einmal einen relativen Wert, sondern gar keinen Wert zu. Die (entwickelte) Modifikation seiner Theorie scheint mir geringfügig zu sein; sie lässt die absolute Sonderstellung des Menschen in Ansehung seiner Vernunft unberührt und betont seine Pflichtenstellung ${ }^{41}$.

Wenn „moralische Selbsterkenntnis... aller menschlichen Weisheit Anfang“ ist, wie Kant meint ${ }^{42}$, so gehört eine Erkenntnis der eigenen Verantwortung für und gegenüber Verantwortungsgegenständen, die nicht-menschlich sind, auch zu dieser moralischen Selbstvergewisserung. Wir können nicht mehr nur die Vernunft an sich als Selbstzweck sehen und alles andere nicht-menschliche Leben oder Sein als bloßen Diener für diese angebliche „Krone der Schöpfung“ ansehen. Die idealistisch-aufklärerische Präpotenz der ausschließlichen Selbsthervorhebung des Vernunftwesens ist einzuschränken. Die Auszeichnung des Menschen als des moralischen, Verantwortung und Verpflichtung tragenden Wesens braucht damit überhaupt nicht aufgegeben zu werden. Wir dürfen auch nicht den haarspalterischen Trick mitmachen, dass der Mensch nur aus ästhetischen oder exemplarisch-erzieherischen Gründen sich der Natur und ihren Arten gegenüber verantwortlich verhalten müsse, um moralisches Verhalten „in Ansehung“ von nicht-menschlichen Kreaturen und ihren ökologischen Systemen zu begründen.

Der Mensch selbst ist Teil der Natur. Ausgezeichnet ist er allerdings dadurch, dass er diese erkennen und zum Teil, d. h. begrenzt, durch ihre Gesetze verändern, in seinem Sinne manipulieren kann (und heutzutage auch muss - schon aus speziesistisch-anthropozentrischen Gründen). Damit nimmt er eine erweiterte Verantwortlichkeit auch für die Natur mit auf sich. Die Natur jedenfalls ist nicht nur für den Menschen da: Dies hat eine abgewogene, faire Beurteilung nach allen Erkenntnissen der biologischen Evolution und der "Schicksalsgemeinschaft" von Menschen und nicht-menschlicher Natur zuzugestehen - wenigstens unter dem Anspruch des repräsentativen, auch anderen Wesen Existenz- und Erhaltungsberechtigung zuerkennenden „moralischen“ Denkens. Der extreme moralische Rigorismus, der die Sittlichkeit ausschließlich in der Selbstachtung, in Gestalt der Achtung vor dem Sittengesetz ohne jede empirische Beimischung sieht, ist ebenso

\footnotetext{
${ }^{41}$ Michael Bartsch, brieflich an den Autor ca. 1981.

${ }^{42}$ Kant, Metaphysik der Sitten, II, $\$ 14,441$.
} 
ein in seiner Härte und Vernunftüberschätzung nicht durchhaltbares Extrempostulat eng aufklärerischer Provenienz, wie es die strikte Trennung von Natur und Geist, von den Bereichen der Notwendigkeit und der Freiheit gewesen ist.

Es zeichnet den Menschen aus, ist m. E. wesentlich ein Teil seiner Menschenwürde, dass er nicht nur für seine auf seine Mitmenschen gerichteten-Handlungen Pflichten übernehmen kann, sondern dass er auch gegenüber anderen lebendigen Naturwesen und für (statt nur ,in Ansehung von“) Natursysteme und Naturwesen und auch für Ökosysteme (bis hin zum recht „verletzlichen“ „blauen“ Heimatplaneten) Verantwortung übernehmen kann und heute sogar muss - bereits aus humanen Eigeninteressen und Klugheitsgründen. Als Teil des Naturganzen, als ein besonders eingriffsmächtiger Akteur hat der Mensch als ein ausgezeichneter Teil der Natur geradezu eine Pars-pro-toto-Verantwortung für das Ganze zu übernehmen. Und spezifisch menschlich ist es auch - macht eben mit seine über einen total anthropozentrischen Chauvinismus hinausgehende Würde aus -, dass er symbolisch-projektiv anderen Wesen moralische Quasirechte, Existenzberechtigung, Erhaltungsberechtigung zuerkennen kann und/oder ohne Gegendienst oder Gegenpflicht-Einforderung Pflichten für sie, gegenüber ihnen auf sich nehmen kann - und soll. Er ist das Wesen, das Einsicht in den Gesamtzusammenhang haben kann und über seine anthropozentrische Beschränkung hinaus dem Gesamtsystem sowie den ökologischen Teilsystemen der Natur als auch lebendigen Partnern in der Natur Existenzberechtigung und Erhaltungsanrechte zuerkennen kann - und soll. Sagesse oblige (Wissen verpflichtet). Diese übergreifende Moral, welche die wechselseitige Angewiesenheit ${ }^{43}$, ja, eine quasi wenn nicht gerade „partnerschaftliche“, so doch rücksichtsvolle, stellvertretende, repräsentativ mitdenkende, „mitsorgende "Beziehung zwischen Mensch und Naturwesen berücksichtigt, erscheint eher dem Menschen würdig, sozusagen „ehr-würdiger“, ja, „humaner “als die traditionelle strikte Selbstbeschränkung auf menschliche Interessen.

\footnotetext{
${ }^{43}$ Selbst von einer „quasi partnerschaftlichen Beziehung“ zwischen Mensch und Natur zu sprechen, ohne die Natur als solche zu personifizieren, erschiene angesichts der essentiellen wechselseitigen Abhängigkeit trotz der Einseitigkeit der Partnerschaft, wenigstens metaphorisch, insofern nicht allzu problematisch, als der Mensch als Teil der Natur - moralisch gesehen - diese ohnehin nicht in jeder Beziehung radikal objektivieren kann. Jede radikale Objektivierung und strikt wissenschaftliche Perspektive stellt eine für empirische Erkenntnis zweifellos notwendige, aber stets auch dem Gesamtleben und seiner gelebten und erlebten Einheit gegenüber eingeschränkte Perspektive dar.
} 
Allem Lebendigen, das einen relativ integrierten Systemzusammenhang und eine Kontinuität im ständigen Austausch mit der Umwelt aufrechterhält, dem in gewisser Weise Selbsterhaltung, Selbstsein und eine funktionale Rolle in einem natürlichen System zuzusprechen ist, könnte also im Sinne einer säkularisierten Ehrfurcht vor der Natur samt ihres Systemzusammenhanges und ihrer Entwicklung und vor einer übergreifenden Einheit des Lebendigen eine QuasiExistenzberechtigung zugesprochen werden. Diese könnte nur begründet bestritten oder abgelehnt werden, sozusagen nicht ohne Not, nicht unnötig - und fallweise meist nur in vertretbaren Einzelfällen.

Lebensberechtigung ist ethisch meist ein Prima-facie-Recht/Quasirecht, das nicht ohne besondere Begründung verletzt werden darf - selbst dann, wenn es sich auf nicht-menschliches Leben bezieht.

Bei unseren Güterabwägungen sind also entweder moralische Quasi-Eigenrechte der natürlichen Wesen zu berücksichtigen oder es ist die Pflicht-RechteSymmetrie aufzugeben. Dies beides muss nicht bedeuten, dass man die Verantwortung dem Lebendigen gegenüber, die aus dem relativen Existenzrecht folgt, nun fast ins Grenzenlose ausdehnt, wie es Schweitzers Ethik der „grenzenlosen Verantwortung allem Lebendigen gegenüber" tat (s. u. II.). -Auch hier gibt es natürlich noch erhebliche Unterschiede in der Einschätzung der Nötigkeit: Vegetarier, Buddhisten, Schweitzer-Anhänger sehen, setzen eben die Grenzen anders. Erweiterte Verantwortung muss sich nicht ins Grenzenlose und damit ins Undurchführbare, letztlich Untragbare ausdehnen. Das mystische Einheitserlebnis des metaphysischen Willens zum Leben (wie schon bei Schopenhauer) muss nicht jeder Verantwortung für alles Lebendige überhaupt untrennbar zugrunde gelegt werden. Die Überdehnung ins Grenzenlose ist weder durchführbar noch geeignet als Basis für eine auf Universalisierbarkeit und Allgemeingültigkeit bedachte Ethik.

Eine ins Grenzenlose geführte Überdehnung wäre übrigens auch Spaemanns Fassung eines kategorischen Imperativs, nämlich „nichts in der Wirklichkeit nur als Mittel“ zu gebrauchen, sondern es stets auch als Zweck oder gar Selbstzweck zu sehen. (Ähnlich übrigens Meyer-Abich: „Jeder ist für alles verantwortlich“ - was ebenfalls methodisch undurchführbar wie unlogisch-unsinnig wäre. $)^{44}$.

\footnotetext{
${ }^{44}$ Beides wäre - streng genommen - sogar eine Ausdehnung weit über Albert Schweitzers Verantwortung oder Mitverantwortung allem Lebendigen gegenüber hinaus.
} 
Vielleicht sollte man diesen Gesichtspunkt auf dynamisch sich im Stoffwechsel erhaltende, „lebendige“ Ganzheiten wie Öko-Systeme einschränken und fordern, dass sie nur begründet, also mit „guten Gründen“ aufgrund eines höher bewerteten Systems, Lebens oder kulturellen Ziels (lediglich) als Mittel benutzt werden können. Dies könnte man auf lebendige Einzelwesen, besonders aber eher noch auf Arten und ökologische Natursysteme beziehen. Güterabwägungen wären aber auch dann immer ins Prinzipielle zu wenden, müssten mit allgemeinen Begründungen arbeiten und dürften nicht ausschließlich anthropozentrisch nur dem menschlichen Einzelinteresse dienen, sondern hätten moralische Existenzund Erhaltungsrechte natürlicher Wesen in sinnvoller Gesamtabwägung zu berücksichtigen.

Von der absoluten anthropozentrischen Charakterisierung der Moral braucht nach allem im Grunde nur zwei Teile aufgegeben zu werden: die Einschränkung der Selbstzwecke auf Vernunftwesen und der Gegenstände moralischer Pflichten auf ausschließlich Personen (Menschen). Die Auszeichnung des Menschen als des moralischen Wesens als des moralischen Adressaten und Akteurs bleibt bestehen. Sie wird sozusagen "fairer“, geradezu „humaner" gegenüber anderen Wesen gestaltet, wenn man diese Begrenzung verabschiedet. In der Tat: die Erweiterung der Eingriffs- und Aktionsmacht des Menschen - insbesondere die ungeheuerlich gewachsene technologische Macht - erweitern Verantwortung und Verpflichtung. Wissen, Können und Macht verpflichten.

Ethisch jedenfalls sollte die traditionelle, ausschließlich an der strengen Kantischen Vernunftphilosophie orientierte Symmetrie von Rechten und Pflichten aufgegeben werden: Nicht „nur wer Pflichten übernimmt, hat auch moralische Rechte“. Der moralischen Auszeichnung des Menschen als des sich selbst verpflichtenden Wesens wird dadurch kein Jota abgestrichen. Im Gegenteil: der Moralität, der moralischen Autonomie und Würde des Menschen steht es gut an, wenn er sich auch für andere, von ihm abhängige Naturwesen und -systeme mit verantwortlich fühlt - und auch so handelt ${ }^{45}$. Gerade dies erhebt ihn hinaus über den anthropomorph(istisch)en Chauvinismus und gattungsmäßigen moralischen Egoismus und zeichnet ihn dennoch spezifisch epistemisch und moralisch aus,

\footnotetext{
${ }^{45}$ Und der Normal-Beurteiler und -Akteur im Alltag tut das ja meist auch - und zwar nicht nur für seine Haustiere.
} 
führt ihn aber zugleich zu einem sich relativ bescheidenden und realistischeren Verhältnis zur Natur und ihren „Wesen“.

Vielleicht lässt sich dieser Gesichtspunkt der moralischen Würde eher als der strikte moralische Anthropozentrismus vereinen auch mit einer leicht modifizierten Version von Kants berühmten Doppel-„Beschluss“ seiner Kritik der praktischen Vernunft: Das Bewusstsein des „moralischen Gesetzes in mir“ „erhebt... meinen Wert“ (und meine Würde! - H.L.) „als einer Intelligenz, unendlich durch meine Persönlichkeit, in welcher das moralische Gesetz mir" eine besonders humane Verantwortung und Verpflichtung gegenüber bzw. für die Natur, ihre Tiere und lebenden System zumutet ${ }^{46}$.

Es müsste übrigens gerade im Sinne Kants ein echtes Zeichen einer von egoistischen-empirischen Antrieben freien Moralität sein, auch über den kollektiven gattungsmäßigen Egoismus des Menschen hinaus zu gehen, für andere potentiell betroffene Wesen und deren Existenz mitzudenken ${ }^{47}$. Denkt man ethisch wirklich repräsentativ, so müsste man den Imperativ der Pflicht konsequent - soweit mit der Eigenexistenz und der Menschheitserhaltung verträglich - auf „die Natur“ und besonders die lebenden Wesen in ihr ausdehnen. Ohne die Natur „moralisieren“, moralisch erziehen zu können, kann bzw. soll der Mensch „moralisch repräsentativ“im Sinne des Sittengesetzes handeln - auch für andere von seinem Handeln und seiner Macht (auch der Macht der Unterlassung) betroffene Menschen und Naturwesen Verantwortung übernehmen. Über den gattungsmäßigen Egoismus hinaus erhebt gerade auch das seine moralische Würde.

${ }^{46}$ Kant, Kritik der praktischen Vernunft, in Werke, TB-Akademie-Textausgabe, Bd. V (Berlin: de Gruyter, 1968), 162. Das im Text kursiv Hervorgehobene steht abgewandelt für Kants Formulierung „ein von der Tierheit und selbst von der ganzen Sinnenwelt unabhängiges Leben offenbart“.

${ }^{47}$ „Denke repräsentativ!“ (so, „als ob die Maxime deiner Handlung durch deinen Willen zum allgemeinen Naturgesetze werden sollte“(Kant, Grundlegung zur Metaphysik der Sitten, 421), 421, war ja eine von Kants Formulierungen des Kategorischen Imperativs, wobei sogar „Natur“ im allgemeinsten Verstande (der Form nach) gemeint ist, „d. i. das Dasein der Dinge, sofern es nach allgemeinen Gesetzen bestimmt ist“" (ebd.). 


\title{
II. Albert Schweitzer als Pionier der Bioethik und als Öko- und Klimaethiker avant la lettre
}

Albert Schweitzer schilderte sein Visionserlebnis zur Ethik der „Ehrfurcht vor dem Leben“ im September 1915 auf einer Pirogen-Fahrt auf dem Ogowe, als er grübelnd um ein elementares Grundprinzip des Ethischen „ringend“, plötzlich jenes Wort der stets zu beachtenden „Ehrfurcht vor dem Leben“ (dem Willen zum Leben in jeglicher Kreatur einfiel:

\begin{abstract}
Am Abend des dritten Tages, als wir bei Sonnenuntergang gerade durch eine Herde Nilpferde hindurchfuhren, stand urplötzlich, von mir nicht geahnt ${ }^{48}$ und nicht gesucht, das Wort ,Ehrfurcht vor dem Leben“ vor mir. Das eiserne Tor hatte nachgegeben; der Pfad im Dickicht war sichtbar geworden. Nun war ich zu der Idee vorgedrungen, in der Welt- und Lebensbejahung und Ethik miteinander enthalten sind. Nun wusste ich, dass die Weltanschauung ethischer Welt- und Lebensbejahung samt ihren Kulturidealen im Denken begründet ist ${ }^{49}$.
\end{abstract}

Noch Jahrzehnte lang - bis gegen Ende des Zweiten Weltkrieges - hat Schweitzer sich, in unermüdlichen Neuansätzen die Tropennächte hindurch Blätter um Blätter beschreibend, darum bemüht, diese ethische Vision auszubauen. Er wollte widerspruchsfrei die ,ins Grenzenlose erweiterte Verantwortung gegen alles, was lebt“, und die ethische Kluft zwischen der Naturphilosophie („Zwiespalt der Natur", Grausamkeit, Konflikte, Fressen- und Gefressenwerden), dem vorfindlichen „Gesetz des Dschungels“ in der Natur, und den Forderungen der Moral, die wir in der Natur nicht finden können, in ein rationalistisch begründetes Gesamtkonzept zu bringen, die Ethik aus einem obersten Prinzip herzuleiten und dieses Prinzip vernünftig aus der Natureinordnung zu begründen. Die Erkenntnis und unmittelbare Respektierung des Willens zum Leben in mir, „nötigt mir“ (und uns allen) aus Überlegungen zur Gleichberechtigung auf, auch allen anderen Lebewesen und dem in ihnen wirksamen Willen zum Leben die gleiche Achtung

${ }^{48}$ Allerdings hatte er selber bereits in den Straßburger Vorlesungen am 3. 2.1912 wörtlich diese Formulierung verwendet. (Angeblich hatte der Radikalethiker und Vegetarier Magnus Schwantje bereits seit 1902 diese Formel benutzt - allerdings in gänzlich anderer Zuspitzung [Heike Baranzke, „Was bedeutet ,Ehrfurcht' in Albert Schweitzers Verantwortungsethik?", Synthesis philosophica 2012, issue 27: 7-29]).

${ }^{49}$ Albert Schweitzer, Aus meinem Leben und Denken (Hamburg: F. Meiner, 1980), 132. 
und Ehrfurcht entgegenzubringen und allen Wesen (v. a. in meinem konkreten Einfluss- bzw. Handlungsbereich ${ }^{50}$ ) diese Ehrfurcht auch in der praktischen Tat zu erweisen.

Doch Schweitzers Vorhaben blieb ein Torso - aus äußeren wie aus inneren Gründen. Äußere Gründe waren, dass Schweitzer sich nach den Bombenabwürfen von Hiroshima und Nagasaki einem intellektuellen öffentlichen Feldzug gegen die Atomwaffen und die atmosphärischen Nuklearversuche widmete. Da er sich erst intensiv über entsprechende atomwissenschaftliche Grundlagen und Effekte sowie Feldwirkungen kundig machen musste, blieb offenbar keine Zeit für die ethische Theorie mehr ${ }^{51}$.

Doch auch innere Gründe waren dafür wohl die entscheidenden für die Integrationsschwierigkeiten: Schweitzer wollte sich nicht zu Rangordnungen der Lebenswerte verstehen: Jedes Leben war ihm theoretisch - wenn auch nicht praktisch! - gleich heilig. So empfand er sich als „Mörder von Millionen von Bakterien“, um einen Menschen zu retten - und er rettete als Arzt Tausende von tödlichen Krankheiten der Tropen... Er verabreichte dem Pelikan mit tiefen Gewissensbissen die täglichen Fische - unter Verletzung der grundlegenden Gleichberechtigung allen Lebens... Nicht einmal eine Eins-zu-eins-Beziehung ließ sich aufrechterhalten: Viele Lebewesen mussten geopfert werden für die Fütterung eines einzelnen. Doch dieser urprotestantische Schuldkomplex hätte sich durch die Unterscheidung ${ }^{52}$ von unnötigem und lebenspraktisch notwendigem Töten, z.T. wenigstens, bewältigen. Nur unnötiges Töten ist unethisch. Doch sollte das Gewissen sehr wachsam auf die Grenzen der Unnötigkeit achten. („Das gute Gewissen ist eine Erfindung des Teufels“, schrieb Schweitzer bekanntlich gegen jede, zumal

${ }^{50}$ Diese pragmatisch notwendige Einschränkung gestand Schweitzer gegen Ende seines Lebens zu (Albert Schweitzer, Die Weltanschauung der Ehrfurcht vor dem Leben. Kulturphilosophie III, hrsg. von Claus Günzler, Johann Zürcher. 2 Bde. München: Beck, 1999; vgl. z. B. a. Hans Reiner, „Die Zukunft der Ethik Albert Schweitzers“, Journal of Value Inquiry 1968, issue 2: 157-162).

${ }^{51}$ Ohnehin waren seine Ethiknotizen, die sich zu über 2000 Seiten philosophischer und über 1000 theologischer Kommentare und Entwürfe aufhäuften, in einer kaum glaublichen Energie-AusdauerLeistung dem mörderischen Klima, dem enervierenden und alles fordernden Gesamttageswerk des praktischen Urwaldarztes, ständigen Baumeisters, Aufsehers, Gelderwerbers usw. sowie dem Schreibkrampf seiner Hand abgetrotzt! (Schweitzer, Die Weltanschauung der Ehrfurcht vor dem Leben).

${ }^{52}$ Reiner („Die Zukunft der Ethik Albert Schweitzers“: 162) sieht „in der mangelnden Unterscheidung“ zweier „Schuldbegriffe“ den Grund hierfür: der „Schuld aufgrund ungewollter „bedauerlicher“ Verursachung vom „Bestehen „ oder „Eintreten“ eines „Übels“ einerseits und dem „Tun“ von sittlich „Verwerflichem“. 
jede schnellfertige Selbstberuhigung). Doch wo sind die Grenzen? Sollen wir, müssten wir nicht alle Vegetarier werden ${ }^{53}$.

„Leben fördern ist gut“, ebenso Leben vermehren? Unnötig Leben behindern, schädigen, vernichten ist böse für Schweitzer. Noch in ethischen Predigten ca. 1920 (veröffentlicht 1974 unter dem Titel Was sollen wir tun?) forderte Schweitzer dazu auf, möglichst viele Menschen, möglichst viel Leben auf der Erde zu erzeugen - konsequent nach seinem Prinzip. Doch die ökologischen und ökonomischen Widersprüche, Dilemmata, Konflikte ${ }^{54}$ und Fernfolgen einer konsequenten Überbevölkerungs- und Lebensvermehrungsstrategie sah und diskutierte der erste Bioethiker dieses 20. Jahrhunderts damals noch nicht. Der Bioethiker war nur recht begrenzt schon Ökoethiker. Drastische Einschränkungen oder Abänderungen erwiesen sich viel später als nötig, wären aber (auch heute noch) möglich. - Ich bin jedoch überzeugt, dass Schweitzer seine Bioethik heute folgerichtig global zu einer ethischen Berücksichtigung des Ökosystems Erde und auch zu einer Klimaethik erweitert hätte. (Sein Einsatz gegen die atmosphärischen Nuklearversuche und die entsprechenden Kontaminationen gegen Ende der vierziger Jahre zeigt das deutlich).

Auch einzelne Begriffe lassen sich kritisieren, z.B. der von Schopenhauer und Nietzsche verwendete Begriff des „Willens zum Leben“, den Schweitzer aus von diesen und aus der lebensphilosophischen Tradition übernahm; diese beeinflusste ihn mehr, als er es sich eingestand. Der Willensbegriff ist eine ,anthropomorphe" Konstruktion, ein human-analoges Interpretationskonstrukt, das undefiniert, unverändert (in derselben Bedeutung wie beim Menschen) wohl nicht so einfach auf andere Naturwesen - geschweige denn auf Ökosysteme - angewendet werden kann. „Wille“ setzt z.B. Bewusstheit und Zielorientiertheit voraus. (Die von Schweitzer konstatierte ,Selbstentzweiung des Willens zum Leben` in der Natur, die für ihn das schockierende, entrüstende, ethikprovozierende Erlebnis be-

\footnotetext{
${ }^{53}$ Wir Menschen könnten dies durchaus - auch nach Schweitzer: Die Jains und Buddhisten (mancher Denominationen) exerzieren es uns seit Jahrtausenden vor. Und auch die Welternährung wäre auf pflanzlicher Basis derzeit und künftig eigentlich besser zu sichern: Tierische Eiweißproduktion ist viel zu aufwendig, was die pflanzliche Grundlage der Aufzucht und Zusatzfütterung betrifft.

${ }^{54}$ Die Dramatik der Überbevölkerungs- und Ernährungsprobleme drängte sich zu jener Zeit dem denkenden Ethiker noch nicht so deutlich auf wie heute. Was aber sollte sein Ehrfurchtsprinzip für eine notwendige Beschränkung der Bevölkerungslawine leisten können?
} 
deutet, würde zudem, wörtlich verstanden, eine vorgängige oder gar ursprüngliche Einheit dieses Willens - gar in ethischem oder ethik-analogen Sinne? - voraussetzen; diese Einheit dürfte es nie gegeben haben, wäre allenfalls mythisch in religiöser Deutung zu unterstellen, kann jedenfalls von der Philosophie weder hypostasiert noch begründet werden).

Die von Schweitzer theoretisch geforderte Erweiterung der Verantwortung gegenüber allem Leben ist faktisch kaum umfassend durchführbar, wirkt methodologisch geradezu unsinnig: Wer grenzenlos gegenüber allem verantwortlich ist, ist niemandem gegenüber wirklich spezifisch verantwortlich. Unbegrenzte Verantwortlichkeit, nach Grad und Adressatenkreis uneingeschränkt, ist nicht greifbar, ist nicht praktikabel, nicht wirklich handlungsbindend oder verpflichtend. Wer leicht, allzu leicht wie der Verbaltrick eines Politikers „die volle Verantwortlichkeit“ für alles deklarativ übernimmt, besser: zu übernehmen behauptet, übernimmt keine kontrollierbare, keine abgrenzbare, keine „definierbare“ Verantwortung, oder er „übernimmt“ sich total in verbaler Kraftmeierei, plakativer Verantwortlichkeitsmeierei. - Schweitzer kann das so nicht gemeint haben - und deshalb wohl schränkte er später, besonders in seinen unveröffentlichten Ethiknotizen die Herausforderung, die Aktivierung der Verantwortung jeweils auf das Leben ein, das je in „meinen Handlungsbereich“ tritt. Dies ist eine sehr sinnvolle, ja nötige Einschränkung.

Manchmal formuliert Schweitzer so, als soll der ethische Geist der Natur angesichts deren „Selbstentzweiung“ Ethik lehren. Aber ebenso wenig wie man die sozialistische Großutopie der Menschenverbesserung à la Herbert Marcuse auf die Vertreibung des Ausbeutungswillens, des „Kapitalismus“, aus der Natur erweitern kann (damit der „große nicht mehr den kleinen Fisch“ fresse!), so wenig lässt sich der Natur Ethik beibringen oder predigen, so sehr Schweitzers Ansatz zu einer rationalen Begründung einer Naturverehrung nach Franciscus von Assisi beim „Brudergeschöpf Tier“ tendieren mag. (Beiden Ethikern war die mystische Identifikation mit dem anderen Leben das höchste Erlebnisziel, das nach Schweitzer freilich nur in der Hingabe-Ethik des menschlichen Handelns wirklich werden kann).

Selbst wenn wir in der Naturverantwortung sozusagen symbolhaft und exemplarisch Ethik erweitern (unser ethisches Handeln) und beachten, ein Beispiel zu setzen, geschieht das allein in menschlicher, „humaner“ Interpreta- 
tion. Jegliche Projektion einer Ethik in die Natur ist unerlaubt, wäre bliebe geradezu „unerhört“. Ethik ist Ethik des Menschen, ist Menschenwerk, ist unaufgebbar anthropozentrisch als Produkt und Konzeption. Dies gilt selbst dann, wenn wir die Gegenstände der ethischen Rücksichtnahme und die Adressaten der Verantwortlichkeit (die stets nur unsere Verantwortung umfasst) auf andere Wesen - Naturwesen wie höhere Tiere, alle leidensfähigen Kreaturen, ja, auf gefährdete Arten (besonders aktuell!) und gar auf Ökosysteme - ausdehnen wollen. Das gilt auch, wenn wir diesen Arten und Systemen - und nicht nur den Haustieren und Zuchttieren - verantwortlich Sorge, Leidensabwendung oder -minderung, Zuwendung und vielleicht gar einen moralischen Rechts- oder Quasirechtsstatus zuerteilen. Diese moralischen „Quasirechte“(s.o.) sind natürlich nur stellvertretend durch andere, durch Fürsprecher, Ombudsleute, Naturschutzbeauftragte einzufordern: Ähnlich wie man einen Schutzbeauftragen für künftige (Human)Generationen einrichten könnte - etwa wie Naturschutzbeauftragte beim Parlament - könnte auch der Tierschutzbeauftragte im Sinne einer nötigen ethischen Erweiterung des Tierschutzgesetzes tätig werden. Dessen Neufassung sieht immerhin die Tiere nicht mehr als bloße „Sachen“ an (s.o.), aber behandelt sie dennoch in der Praxis der Massenhaltung und Transporte meist weiterhin so!). (In der Frage der Genehmigungspflicht für Tierexperimente geschieht freilich gegenwärtig schon einiges. Bei der Massentierhaltung/Transporte und dem Problem des Artensterbens ist noch viel zu leisten - und zwar recht dringlich!).

Schweitzers Tierethik als Teil seiner Bioethik, die z.T. auch umweltethische Ansätze umfasst, wie Günzler ${ }^{55}$ nachgewiesen hat, enthält solche Forderungen bereits. Schweitzer war Humanethiker, Tierethiker, Bioethiker z.T. Umweltund Ökoethiker avant la lettre, ohne die zentrale Humanität vernachlässigt zu haben ${ }^{56}$.

${ }^{55}$ Claus Günzler, „Ehrfurcht vor dem Leben - Albert Schweitzers Ethik als Grundimpuls für die Umwelterziehung“. In Claus Günzler et al., Ethik und Erziehung (Stuttgart-Berlin-Köln-Mainz: Steiner, 1988), 171-199; Claus Günzler, „Ehrfurchtsethik und Umwelterziehung. Zur pädagogischen Fruchtbarkeit der Schweitzerschen Ethik“. In Claus Günzler et al. (Hg.), Albert Schweitzer heute. (Tübingen: Katzmann, 1990), 110-124.

${ }^{56}$ Auch hier gab es natürlich ethische Vorläufer: nicht nur die pathozentrischen (am Kriterium der Leidensfähigkeit orientierten) Bioethiker nach J. Bentham, sondern z. B. etwa Martensen [Hans Lassen Martensen, Die christliche Ethik (Dän. Orig. 1871-1878), 2 Bd. Karlsruhe, 1887] und Leffingwell [Albert Leffingwell, „An ethical basis for humanity to animals”, Arena 10 (1894)], die in ihrer Ethik einen „humanen Umgang“ zumal auch mit Tieren forderten. 
Der Mensch ist, wie bereits oben erörtert, das (ethische) Verantwortung tragende, das einzige bewusst der Verantwortlichkeit für andere Human- und Naturwesen fähige Wesen. Das zeichnet ihn auch dann ethisch einzigartig aus, wenn wir die herkömmliche, auch bei Kant noch ausdrücklich vertretene Symmetrie von Pflichten und Rechten aufgeben müssen (s. o.) Es mindert die ethische Sonderstellung des Menschen keineswegs, wenn die Gegenstände der Verantwortlichkeit und Fürsorge auf nichtmenschliche Wesen erweitert werden. (Allerdings wäre die totale Pauschalisierung mancher Ökoethiker und neuer selbsternannter Naturfriedens-Aposte ${ }^{57}$ praktisch wie theoretisch ebenso unmöglich wie die ins Grenzenlose überdehnte Verantwortlichkeit).

Übrigens: Wenn Schweitzer in Einzelanalysen und auch bei Besprechungen der eigenen Motivation für seine Entscheidung, die wissenschaftliche, künstlerische und Predigerkarriere zugunsten seines Afrikaeinsatzes aufzugeben, meint, unter dem Signum der „persönlichen Verantwortung“ müsse jeder „etwas von seiner Menschlichkeit opfern“, sich z.B. helfend anderen widmen ${ }^{58}$, so verwendet auch er zumindest implizit noch andere Prinzipien der ethischen Beurteilung als das Ehrfurchtsprinzip allein. So hat beispielsweise der Phänomenologie-Historiker Herbert Spiegelberg bei Schweitzer noch ein weiteres ethisches Grundprinzip, besser: eine Grundnorm, in Gestalt der Weisung „Glück verpflichtet!“ aufgespürt. Dies dürfte richtig sein.

Schweitzer scheint aber implizit noch weitere Normen, z.T. auch solche, die sein Grundprinzip differenzieren, in Anspruch zu nehmen: z.B. das Prinzip umfassender und auf die Schonung und Behandlung aller Kreaturen erweiterte Humanität. Er versteht „das Humanitätsideal“ als „das ethische Grundelement wahrer Kultur“ und fordert, dass „der neue Mensch sich nicht als Herr, sondern als Bruder der Geschöpfe anzusehen habe“ und „von der Unvollständigkeit zur vollständigen Humanitätsgesinnung fortschreiten“ solle ${ }^{59}$.

${ }^{57}$ Meyer-Abich möchte weit über die Schweitzersche Ethik der Ehrfurcht vor allem Lebendigen hinausgehen, wenn er in unüberbietbarer Maximalisierung fordert: „Jeder nimmt auf alles Rücksicht!“ Das ist ebenso unsinnig und undurchführbar wie die Verantwortlichkeit jedes Menschen für alles auf der Welt - eine Idee, die übrigens auch schon - vom Computerkritiker und einstigen Informatiker Joseph Weizenbaum - ausgesprochen worden ist. Verbale Wortmeiereien, die zu nichts mehr taugen, weil sie zu allem eben bloß verbal verpflichten würden.

${ }^{58}$ Albert Schweitzer, Kultur und Ethik, 2. Aufl. (München: Beck 1960), 347.

${ }^{59}$ Albert Schweitzer, Gesammelte Werke, Bd. 5 (München: Beck, o. J.), 170. 
Wenn Schweitzer etwa weiterhin ${ }^{60}$ fordert, man solle das Leben ,in jeder Hinsicht auf seinen höchsten Wert zu bringen suchen", so ist auch hier doch - in Abkehr von seiner Gleichrangigkeitsthese - eine individuen- oder artinterne Wertvergleichung, eine Wertabstufung oder Wertrangfolge unterstellt, jedenfalls eine Wertmaxime in Anspruch genommen. Warum sollte man dann nicht auch Abstufungen, zwischenartliche Wertvergleiche zulassen, und sei es nur, um die erwähnten urprotestantischen Schuldkomplexregungen („Mörder von Bakterien“) zu vermeiden.

Weshalb sollte man dann nicht doch Wertabstufungen ${ }^{61}$ zulassen; nicht etwa - das war ja Schweitzers Sorge! -, um das Gewissen abzustumpfen, eine Art oder ein Ausmaß des „guten Gewissens“ (der „Erfindung des Teufels“) zuzugestehen, sondern um die Kluft zwischen der abstrakt-ethischen Forderung des Ehrfurchtsprinzips und der Praxis etwas einzuengen? Der theoretischen normativen Grundforderung, der Grundnorm, können doch durchaus auch gewisse Praxisregeln (die etwa Dieter Birnbacher für die Zukunftsethik gegenüber künftigen Generationen einführte) zur Seite stehen. Schweitzer weigerte sich - unnötig überkonsequent und somit zugleich unnötig praxisfern -, Regelungen der praktischen Entscheidung vorzusehen. Bringt man aber Praxisregeln ein, macht die Ethik greifbarer, pragmatisch zugänglicher, so bedeutet das keineswegs, dass die Verantwortung beschnitten, die Entscheidung jemandem abgenommen würde. Allenfalls würde sie erleichtert, weil in der Richtungsangabe, Orientierung oder Anleitung vorgelenkt. Die Entscheidung treffen müssen wir stets selbst. Darin ja besteht zum guten Teil unsere Freiheit und auch die Würde, Aufgabe und Größe unserer Verantwortung.

Wenn man bei den Zugeständnissen an Varianten und Zusätzen zur Schweitzer-Ethik soweit gekommen ist, wäre es sinnvoll, auch andere ethische Grundnormen mit zu berücksichtigen und in ein integriertes Ethikkonzept einzubeziehen. Warum, weshalb, wieso soll nur ein einziges oberstes Prinzip herhalten, um alles Ethische daraus abzuleiten oder nur zu rechtfertigen, zu begründen? (Darüber unten mehr). Man könnte also traditionelle ethische Prinzipien dem obersten Grundsatz der zu beachtenden Ehrfurcht vor dem Leben nachordnen

\footnotetext{
${ }^{60}$ Schweitzer, Kultur und Ethik, 353.

${ }^{61}$ Schweitzer wollte ja - wie schon oben erwähnt - auch die Menschen ethisch auf ihren „höchsten Wert" bringen.
} 
oder zur Seite stellen - etwa die Prinzipien der fairen Behandlung und Güteraufteilung, Chancenbeteiligung und Gleichberechtigung, der Gerechtigkeit, der Toleranz, der Leistungsgerechtigkeit, der negativen wie der positiven Goldenen Regel („Was du nicht willst, das man dir tu', das füg' auch keinem anderen zu“ - als negative Formulierung dieses Prinzips des Austausches von Handelnden und Betroffenen und als eines Kriteriums, genauer: als einer notwendigen formalen Bedingung, ethischen Handelns bzw. der entsprechenden Beurteilung). Das Kantische Universalisierbarkeitsprinzip, zumal den Kategorischen Imperativ anerkennt bzw. benutzt Schweitzer ja selber öfters - und zudem gegen Ende seines Lebens neben dem Ehrfurchtsprinzip ein über alle Formalprinzipien und Kriterien hinausgehendes Wohlwollens-Prinzip bzw. das Humanitätsprinzip gegenüber (zunächst mir nicht gefährlichen) Mitlebewesen, ein Solidaritäts- und Mitleidsprinzip, das sich auf alle in meinen Handlungs- und Erfahrungsbereich kommendes mitlebende und (mit)leidende Wesen beziehen mag. Schweitzers Ethik ließe sich anreichern, vervollständigen und praxisnäher formieren, wenn man solche Ergänzungen vornähme und Praxisregeln einführte. Sie würde dann freilich von ihrer eindrucksgebietenden Einfachheit, Höchstforderung und Monumentalität verlieren.

Normative Ethik ist Aufgabe, ist stets erst als Menschenwerk zu sehen, zu verbessern, zu entwerfen, zu entwickeln. Sie ist keine Angelegenheit einer reinen und bloßen Erkenntnis, einer Ableitung gar aus theoretischem Wissen allein, oder gar aus von Schweitzer so betonten „denknotwendigen“ Grundsätzen oder Grundlagen. Die Tradition - bis noch zu Kant - hat die Ethik zu sehr zugleich am Muster der theoretischen Wissenschaft und am Vorbild des Rechts zu begreifen versucht. Bestimmte Fallen halten beide Modelle bereit. Und vom traditionellen Letztbegründungs- und Deduktionsrationalismus waren alle diese Versuche (auch Schweitzers Ansatz!) noch geprägt, wenn sie den Allgemeinheitsanspruch als ableitbare generelle Gültigkeit kraft theoretischer Grundlage, als „Objektivität: in Analogie zur kognitiven theoretischen Wissenschaft verstanden ${ }^{62}$.

${ }^{62}$ Manche wollten diese Objektivität gar naturalistisch legitimieren: Naturalistische und gar empiristische Ethikbegründungen gab es immer wieder in der Geschichte - bis hin zur Nutzung der Soziobiologie für ethische Begründungszwecke. Ähnliche Fallstricke bieten sich freilich auch den intuitionistischen ethischen Fundierungsversuchen, die ebenso kognitivistisch ethische Werte und Normen aus einer eigenen, nichtnatürlichen Wertintuition, Werterkenntnis, Wertkognition begründen wollen. 
Andere - wie Schopenhauer - wollten die Ethik allein auf Emotionen, Gefühle, (Mitleids-) Erleben stützen - auf deren empirische Gegebenheit oder das mitleidende Mitempfinden in projektiver und eventuell generalisierender Identifikation mit fremdem Leiden oder - positiv - mit Lebensfreude, Lebenslust und deren projizierter Berechtigung ${ }^{63}$. Aus rein kognitiv-theoretischen Voraussetzungen lässt sich aber (außer in den trivialen Fällen bloßer logischer Abschwächung etwa durch Hinzufügung eines beliebigen normativen Satzes mit Hilfe der OderVerbindung) kein Sollens-Satz, keine Norm herleiten.

Eine Art von sog. naturalistischem (besser: „normativistischem“) Fehlschluss weist auch Schweitzers Versuch auf, aus der Feststellung, ein meinem gleicher "Wille zum Leben“ sei in anderem Leben gegeben, nun die normative Forderung zu deduzieren, diesem „Willen zum Leben“ sei Ehrfurcht, Achtung entgegenzubringen, wie ich sie auch dem „Willen zum Leben“ in mir selber entgegenbringe(n soll) ${ }^{64}$. Entweder wird ein anderes generelles Prinzip wie das erwähnte Austauschprinzip von Handelnden und Betroffenen von vorneherein auf eine normative Generalforderung angewandt, oder das allgemeine Ehrfurchtsprinzip wird als Norm bereits in Anspruch genommen (also ein Zirkelargument vollführt), oder man erschließt aus einer Tatsachenfeststellung (oder besser: Tatsachenunterstellung, -interpretation) die normative Forderung (und begeht direkt den normativistischen Fehlschluss), oder der Schluss wird aus einer mystischen Erlebnisidentifikation mit dem fremden oder dem allgemeinen „Willen zum Leben“ gezogen - mit ähnlicher Fehlerhaftigkeit. Ethische Verallgemeinerungen und Objektivität (besser: „Objektivierungen“) sind von uns konzipiert, geschaffen vom sinn- und normsetzenden Menschen; sie sind nicht durch Deduktion quasinormativ zu gewinnen!

${ }^{63}$ Alle diese Begründungsversuche verfallen zudem einer „normative fallacy“ einem dem naturalistischen Fehlschluss nach George Edward Moore entsprechenden Falschargument, das vom Sein wesentlich aufs Sollen, von kognitiv-theoretischen Erkenntnissen auf die Annahme und Verpflichtung einer Norm, einer normativen Forderung schließt.

${ }^{64}$ Ethische Forderungen, Normen, Regeln, Prinzipien sind - als universalmoralische verstanden (i.w. Sinne des Ausdrucks „Ethik“ als „normative Ethik“ - hier nicht als bloße theoretische oder philosophische Disziplin oder gar als auf Sprachgebrauchsanalysen eingeschränkte Metaethik!) - ethische Regeln sind normativ, schreiben vor, werten, beurteilen. Sie sind nicht aus bloß kognitiv beschreibenden Grundlagen zu deduzieren. Normatives ist nicht aus rein Deskriptivem abzuleiten, ist nicht aus deskriptiven Prämissen allein zu begründen, geschweige denn als handlungsleitend zu legitimieren. 
Verwandt ist dieser fehlverstandene kognitive Legitimationsanspruch durch Deduktion freilich auch der Tradition des Rationalismus, der Letztrechtfertigungsansprüche, die aus einem obersten Grundsatz gleichsam axiomatisch alle Folgerungen (hier also Normen) zu beweisen oder zu deduzieren versuchen. Die Suche nach dem obersten, dem sicheren, unbezweifelbaren Prinzip der Ethik war zwar theoretisch das Leitmotiv von Schweitzers Ethikfundierung. Er war und blieb zugestandenermaßen Aufklärer und Rationalist, der die Ethik „denknotwendig" begründen wollte (was immer dieses jenseits dieser logischen Tautologie bedeuten sollte). Und das, obwohl er letztlich - nach der untergründig von ihm wohl als unmöglich, also als erfolglos bewerteten Letztrechtfertigung durch Logik - beim mystischen Erleben einer Solidar- und Emotionseinheit mit allem „Willen zum Leben“ endete! Schweitzer war dem traditionellen rationalistischen Denken verhaftet, konnte die theoretischen und methodologischen Schwierigkeiten, die dem Letztbegründungsrationalismus aus den Entwicklungen der analytischen Philosophie und Wissenschaftstheorie dieses Jahrhunderts erwachsen würden, wohl noch nicht vorwegnehmen. Es entbehrt nicht einer gewissen Tragik, dass er auf ein Pferd setzen musste, das sich als Pegasus, als Fiktion erwies. Hätte er von dem generell notwendigen Scheitern des absoluten Letztbegründungsrationalismus gewusst, wie es der Kritische Rationalismus Karl Poppers und seiner Nachfolger erwies, so hätte er nicht unnötigerweise noch der Fiktion des obersten Deduktionsprinzips nachlaufen und dieses als einziges finden, etablieren und deduktiv zu sichern versuchen müssen. Er hätte die erwähnten unabhängigen weiteren Prinzipien hinzunehmen und seine Ethik praxisnäher gestalten können. Er hätte auch nicht den „Zwang“ zur deduktiven Gewinnung von Normen aus deskriptiven Prinzipien oder aus einer „Naturphilosophie“ nachgeben müssen, hätte nicht durch Umschreibungen und etwas undurchsichtige Übergänge die Kluft zwischen Natur und Moral, zwischen Natureinsicht und moralischer Einstellung scheinbar überbrücken, den ethischen Grundzwiespalt irgendwie „eskamotieren“ müssen. Schweitzers menschlich so beeindruckender Versuch, die Ethik so bloß „theoretisch“ rationalistisch als „denknotwendig“ neu zu begründen, blieb auch aus theoretischen Gründen ein Torso. Und er musste es bleiben. Der Autor selber muss dies untergründig geahnt haben. Vielleicht hoffte er noch darauf, einen plötzlichen intuitiven Durchbruch durch sein geradezu monomanisch wiederholtes ständiges Neubeginnen herbeizuführen, durch immer wieder ansetzendes Neuschreiben die Erleuchtung, die erlösende Idee herbeizwingen zu können, 
die es ihm gestatten würde, die erahnten, in tiefem Grunde "gewussten“ oder wenigstens richtig geahnten theoretischen Schwierigkeiten zwischen Naturphilosophie und Moralbegründung, zwischen rationalistischer Deduktion und normativer Allgemeingültigkeit, zwischen Kognitivismus und Normativismus, zwischen Rationalität und „mystischem“ Teilhabe-Erleben zu lösen. Es war ihm nicht vergönnt, ja, konnte schon aus inneren Konsistenzgründen nicht gelingen.

Seine geradezu geniale ethische Intuition, praktisch quasi-„,mystisch“ ausgelöst, schien ihm zu Recht überzeugend und leitete ihn sicher im alltäglichen Handeln, bei seiner so bewundernswerten Lebensgestaltung - in wahrhaft imponierender Konsequenz. Überkommene, überzogene rationalistische Ansprüche an materiale Letztbegründungsargumente, ein übertriebenes herkömmliches Vertrauen auf die Erkenntniserzeugungskraft rein logischen Denkens ließen ihn - nahezu notwendig - in eine „rationalistische Falle“ übersteigerter Erkenntnisgewinnungs- und -sicherungs-Ansprüche geraten.

Im Handeln, in der ethischen Praxis ließ er sich durch dieses bloß theoretische Scheitern, das ihm bewusst gewesen sein, das ihn bedrohlich erscheinen musste, nicht beirren. Unbeirrbar ging er seinen Weg - das ethische Genie der humanen Praxis. Die Theorie vermochte er letztlich nicht konsistent zu integrieren. - Aber wer überhaupt vermöchte das schon?

Schweitzer war ein Genie der humanen, der ethischen Praxis. Er mühte sich zwar, seiner eigentlichen Berufung, die er fühlte, zu folgen: Philosoph zu sein - oder besser: sein zu wollen, doch konnte er neben den fast übermenschlichen Ansprüchen seiner ethischen Praxis nicht auch noch einer der größten Theoretiker der Ethik sein. Selbst ein Schweitzer ist ein endliches, ein wie alle auch begrenztes, in Fähigkeiten und Leistungen beschränktes Wesen - so unglaublich, ungeheuerlich, so unbeschreiblich groß seine einzigartig vielfältigen und breiten Leistungen und Talente auf den unterschiedlichsten Gebieten der Praxis, Kunst und Wissenschaft sowie der Inkarnation tätiger Christlichkeit auch waren. Als Theoretiker fand er zu sehr treffsicheren, scharfsichtigen Urteilen über fast alle ethischen Entwürfe aller Hochkulturen, Einschätzungen und Bewertungen, die sich in bemerkenswerter Kondensation auf das Wesentliche konzentrierten. Er konnte und wollte einfach und "tief“" zugleich denken, die Grundstrukturen ethischen Engagements und Denkens mit nachtwandlerischer Sicherheit aufspüren und kritisch namhaft machen. Die Fülle widerstreitender Einsichten, Elemente und Prinzipien in eine konsistente Einheit zu bringen, in ein umfassendes 
Konzept widerspruchsfrei zu integrieren - das vermochte er nicht. Fast etwas tragisch mutet es an, dass er - theoretisch irregeleitet durch falsche, überstiegene, überzogene Ansprüche des herkömmlichen Letztbegründungsrationalismus des einen absoluten obersten Grundsatzes - dahin gedrängt wurde, das Unmögliche möglich zu machen zu wollen. Und diese Unmöglichkeit schien ihm identisch mit der Grundaufgabe der Ethikbegründung. Sie ist es nicht. Hätte er es gewusst oder geahnt, hätte er sich vom Deduktionszwang der rationalistischen Tradition lösen können, dann hätte er wohl sein Hauptziel, eine sinnvolle, widerspruchsfreie und praxisnahe Begründung der Ethik in systematischer Absicht - freilich auf bescheidenerer Ausgangsbasis, was die Begründungsstrenge betrifft - durchaus erreichen können. Jedenfalls hätte er sich nichts eklatant Unmögliches vorgenommen. Er ist und bleibt einer der bedeutendsten Menschen, wie Albert Einstein urteilte. Er ist und bleibt auch ein bedeutender Ethiker, nicht nur der überragende Praktiker der Humanität, sondern auch ein hohes Vorbild ethischer Humanitätsgesinnung, ein Vielseitigkeitsgenie - und auch ein bedeutender Kritiker und Theoretiker der Ethik.

Schweitzer wollte rational hinausgelangen über die durch das tiefe Denken erkannte „Zweispaltung“ der Natur in sich und vor allem zwischen Natur und Ethik. Er hat die angestrebte Re-identifikation mit allem Lebendigen und dem „unendlichen Sein“, letztlich irrational-emotional angenommen als, wie er sagt, „mystische“ Erlebnisphilosophie! Diese wirkt bei Albert Schweitzer wie eine letzte Zuflucht zu einem Halt am sprichwörtlichen Strohhalm, nachdem er teils rational, teils intuitiv wohl gesehen hat, dass der ersehnte rationale Weg zur intellektuellen Rettung nicht trägt. Trotz aller subjektiven Ehrlichkeit des Autors, trotz aller rationalen Begründungsversuche Schweitzers sieht diese Zuflucht, wenn nicht gar nach einem „Credo quia absurdum“, so doch nach einem quasi- rationalistischen Verbrämungsversuch der Sicherung des Unmöglichen aus - oder nach einem versteckten normativistischen Fehlschluss aus dem Schopenhauerisch-Nietzscheschem „Willen zum Leben“. Dieser äußert sich bei Schweitzer freilich nicht als Wille zur Bemächtigung oder gar „Übermächtigung“, sondern als ethische Anerkennung des jeweiligen Lebensdranges, Überlebenswillens usw. Er bedeutet also im Grunde wohl eine fast romantische Identifizierung mit allem Willen zum Leben, ein utopisch-kosmisches Streben zur Vereinigung des Ich mit 
dem ihn umgebenden „unendlichen Sein“, von dem Schweitzer oft schreibt - zumal in seinen Nachlassnotizen zur Ethik ${ }^{65}$.

Ist der Wunsch zur kosmischen Identifizierung oder wenigstens widerspruchslosen Integration in das uns umgebende „Sein“ also doch so etwas wie ein romantisches Heimatstreben? Der Mensch möchte sich in der ihn umgebenden Natur, in der Welt heimisch fühlen, Haltepunkte finden, sich am anderen Leben orientieren, sich selbst als Teil des Lebensstromes verstehen und einrichten können. Schweitzer möchte den Herrschafts- und Machtimpuls à la Nietzsche durch die tiefe Solidarität mit allem Lebendigen ersetzen. Der unterstellte gemeinsame „Lebenswille“, dieses etwas undurchsichtige, zu pauschale Konstrukt der lebensphilosophischen Tradition, soll als Ansatzpunkt einer neuen gesamtheitlichen Einheitsethik auf der Grundlage eines einheitlichen Weltverständnisses herhalten. Schweitzer scheint entgangen zu sein, dass eine solche Begründung der Ethik, wenn man sie ihrer romantischen Identifikationsimpulse entkleidet - und diese sind ihm als einem erklärten Rationalisten Schweitzer eigentlich fremd -, doch auch ein biologistischer oder „lebenswillens-philosophischer“ Fundierungsversuch ist: Schweitzer ist somit übrigens gar nicht so weit von Nietzsche entfernt, was die gesuchte Ausgangsbasis angeht: Er ersetzt Nietzsches Machtstreben durch den Lebenswillen und dessen „ethische“ Anerkennung freilich durch das tiefe Bewusstsein einer den Menschen verpflichtenden Solidarität alles Lebendigen angesichts dieser grundlegenden Gemeinsamkeit, dieser gleichsam „mystisch“ erlebten Einheit(sbestrebung). Schweitzer ist sich nicht im Klaren - jedenfalls eingestandenermaßen und an der argumentativen Oberfläche nicht -, dass kein Folgerungsschritt vom biologischen Fundament (das ja gar nicht zu leugnen ist) zum normativen Impuls der ethischen Anerkennung führt: Kein „naturalistischer“ (besser: normativistischer) Fehlschluss vom Sein (des Lebenswillens) auf das Sollen der „Ehrfurcht vor dem Leben“, auf die Achtungsnorm, trägt rein logisch-rational. Untergründig scheint Schweitzer dies auch zu ahnen - gar zu wissen? Deshalb endet er nach dem Scheitern des rationalen Durchgangs bei seiner „rationalen“ (als Endergebnis der Vernunftbemühungen präsentierten) sog. „Mystik“, einer zwar in der Sphäre einer Erlebensidentifikation formulierten Gemeinschaftlichkeit alles Lebendigen, die sich aber - das ist das Wichtige und Charakteristische bei Schweitzers Vernunftmystik - nicht in passiv erlebten und nur innerlich

\footnotetext{
${ }^{65}$ Schweitzer, Die Weltanschauung der Ehrfurcht vor dem Leben.
} 
kultivierten Identifizierungen, Stimmungen, Haltungen usw. äußert, sondern in aktiver Verpflichtung, in umfassender, ja, unbegrenzter Verantwortlichkeit zur Lebenserhaltung und -erhöhung, kurz: in aktiver Caritas - im Rahmen des je eigenen Handlungsbereichs. Während die traditionelle religiöse Mystik gleichsam erotische Züge trug, ist Schweitzers Vernunftmystik aktiv-caritativ. Sie sollte, um Missverständnisse zu vermeiden, gar nicht „Mystik“ genannt werden. Es handelt sich um eine weltzugewandte, geradezu „weltliche“ "aktive Hinwendungsethik“: Schweitzer spricht ja gegenüber den indischen Entwürfen einer rein innerlichkeitsorientierten Selbstvervollkommnungsethik stets von einer notwendigen „Ethik der aktiven Hingabe“, einer „Hingebungsethik“, die freilich der tieferen Selbstvervollkommnungsethik nichts von ihrem Vorrang nimmt, aber diese doch durch eine charakterische Koppelung bindet: Man könnte geradezu an eine ethische, säkulare Fassung der christlichen Verbindung vom Gottesliebe-Gebot und tätiger Nächstenliebe denken (wie sie in Matth. 22: 37-40 zum Ausdruck kommt). Nur durch aktive ethische Aufgabenerfüllung, durch tätige Hingabe, kann nach Schweitzer die tiefere Selbstvervollkommnung, die ethische Grundforderung verwirklicht werden. Nicht aber schon oder gar allein durch philisterhafte Listenerfüllung von ethischen Aufgaben - bloß als gleichsam notwendig zu erfüllendes Begleitgebot der Selbstvervollkommnungsethik, als deren „notwendiges Übel“ sozusagen. Die Ethik tätiger Hingabe ist ein eigener Pol oder Brennpunkt von ethischer Wesentlichkeit: Schweitzer benutzt vielfach Bilder wie die beiden Brennpunkte einer Ellipse, Hingebungsethik und Selbstvervollkommnungsethik als gleichberechtigte und gleich notwendige, aufeinander verwiesene und verweisende fokale Drehpunkte. Nicht einer von beiden allein könne verabsolutiert werden. Insbesondere kann man wohl nach Schweitzers Ansicht - ohne tätige Hingabe und ohne deren ganz eigene Antriebskraft - nicht die Selbstvervollkommnung verfolgen. Bloßes Selbstvervollkommnungsstreben ohne ethische Aktivität liefe leer, „entweltlichte“, „verpassivierte“ gleichsam den Vervollkommnungsimpuls, ja, entwertete ihn auch in gewissem Sinne als egozentrisch, philisterhaft. Die alleinige instrumentelle Deutung der aktiven Hingabe als eines bloßen Erfüllungsmittels der als überragend angesetzten Selbstvervollkommnungsethik würde ebenfalls als philisterhaft zu verstehen sein.

Dies alles sind sicherlich pragmatisch-ethisch außerordentlich wichtige und weiterführende Einsichten. Sie sind aber schon im normativ-ethischen Be- 
reich zu verorten. Sie sind weder „mystisch“ im überkommenen Sinne, noch führen sie aus der zuvor erwähnten Schwierigkeit des verdeckten normativistischen (fälschlich „naturalistisch“ genannten) Fehlschlusses, der angestrebten Überbrückung zwischen Seinserkenntnis und Sollensbegründung hinaus. Auch können sie nicht dazu dienen, die tiefgründende „Fremdheit“ des Ethischen und des ethischen Menschen in der Naturwelt zu überwinden oder gar zu überspringen.

\section{Zusammenfassende Thesen}

1) Kants Beschränkung der Selbstzwecke auf Personen (Vernunftwesen) ist aufzugeben: Die Dichotomie „Person“: „Sache“ ist unvollständig (neues TierschutzG1998: „Mitgeschöpfe“). (Tierquälerei ist z. B. nicht bloß abstoßend, weil sie für Menschenerziehung ein schlechtes Beispiel gibt, sondern explizit unmoralisch).

2) Tiere, ganze Natursysteme (Ökosysteme), „das Ganze der Natur“, die Öko-Sphäre, ja auch der verletzliche „blaue Planet“ sollten/können nicht bloß als Mittel (Sache) gewertet werden.

3) Die Symmetrie von Rechten und Pflichten ist abzuändern. Manche nichtmenschliche Naturwesen wie sog. „höhere“ Tiere - zumal Primaten wie Menschenaffen - haben (zugeschriebene oder durch Repräsentanten/Ombudsleute wahrzunehmende) moralische Quasirechte, ohne selbst verpflichtet oder verantwortlich gemacht werden zu können. (Auch Haustiere nicht eigentlich!) Tiere sind zwar Adressaten (traditionell fälschlich „Gegenstände“ genannte), aber nicht Träger von Verantwortung. Entsprechende „bedingte“ Quasirechte könnten auch den heute noch nicht existierenden Menschengenerationen (Birnbacher) und künftig zu erhaltenden anderen Spezies zukommen.

4) Anthropozentrismus in der Ethik ist Adressaten-bezogen einzuschränken: erweiterte Verantwortungsethik (z.B. à la Hans Jonas).

5) Dennoch bleibt eine anthropologisch-moralische „Alleinstellung“ der Menschen bestehen: Nur sie können übergreifende Zusammenhänge erkennen und müssten dringend repräsentativ auch für andere Verantwortungs-„Gegenstände “ und -Systeme Erhaltungsverantwortung übernehmen: Wissen, Können und Macht verpflichten/machen uns und nur uns für diese und deren Leben 
bzw. Weiterexistieren (z. B. als Gattung) verantwortlich. Daher betont Schweitzer: „Keiner mache sich die Last seiner Verantwortung leicht..."

6) Über (s)eine „denknotwendige“ rationalistisch- theoretische Ableitung aus dem Lebenswillen hinaus sollte Schweitzers Ethik der „Ehrfurcht vor dem Leben“, der „ins Grenzenlose erweiterte Verantwortung gegen alles, was lebt“, eher als normative Aufgabe und Aufforderung, als Appell an das Einzelgewissen (und an alle!) gedeutet werden (die „Kompassethik“ Schweitzers, die nur die Richtung vorgibt: entscheiden muss jeder selbst).

7) Schweitzers absolute Ablehnung jeglicher allgemeiner Rangordnung der Lebenswerte ist nur so (nämlich als appellartig) zu verstehen und praktisch situationsgebunden und "gewissenhaft“ zu moderieren, wie er selber es einsah und handhabte (Pelikanfütterung mit auch lebenden Fischen, der Mensch und Arzt als „Mörder von Bakterien“, lebensnotwendiger Verzehr von Organismen).

8) Jedoch ist eigentlich gar kein urprotestantischer Schuldkomplex, keine „Übersteigerung des Schuldbewusstseins“ nötig, die sich z.B. ergäbe aus der „mangelnden Unterscheidung“ zwischen einer unvermeidbaren "Schuld“ „bedauerlicherweise“ oder ungewollt eintretender Verursachung einerseits und einem sittlich vorwerfbaren ,verwerflichen Tun“ andererseits (Reiner) - wie alles unnötige Leid- und-Schmerz-Zufügen, gerade auch bei Tieren.

9) Schweitzers Bioethik lässt sich letztlich durchaus in seinem Sinne über die bloß individualethische Perspektive und die notwendige praktische Bindung der konkreten Verantwortung an den eigenen Handlungsbereich hinaus erweitern zu einer umfassenderen Öko-Ethik der (erweiterten) gesellschaftlich-sozialen Verantwortlichkeiten zu einer (direkten und indirekten, auch globalen) Umweltethik (vgl. Schweitzers Protest gegen atmosphärische Nuklearversuche!).

10) An Schweitzers Ehrfurchtsethik lassen sich ethisch-pragmatisch sinnvoll andere traditionell-ethische Konzepte wie Fairness, Gerechtigkeit, Toleranz, Goldene Regel (in negativer wie positiver Fassung!) anschließen - als Ergänzung oder Ausdifferenzierung.

11) Die gesellschaftlich-sozialen und institutionellen Verantwortlichkeiten sollten sich (trotz seiner früheren gegenteiligen Äußerungen) ebenfalls in Schweitzers (stets zu konkretisierender) Auffassung der Ethik als „konkreter 
Humanität ${ }^{\prime 66}$ einbetten oder integrieren lassen, wie er dies am Lebensende (1961 und 1964 in seinem Humanitäts-Vermächtnis) selber umrissen hat: „Die Gesinnung der Humanität hat heute weltgeschichtliche Bedeutung“"

12) Im Zweifel immer für konkrete Humanität - auch für die anderen Lebewesen und Ökosysteme!

In dubio pro humanitate practica/concreta - etiam pro creaturis et in systemis.

\section{Literaturverzeichnis}

Baranzke, Heike. „Was bedeutet ,Ehrfurcht' in Albert Schweitzers Verantwortungsethik?“, Synthesis philosophica 2012, issue 27: 7-29.

Birnbacher, Dieter (Hg.). Ökologie und Ethik. Stuttgart: Reclam 1980.

Birnbacher, Dieter.: Verantwortung für zukünftige Generationen. Stuttgart 1988.

Birnbacher, Dieter.: Klimaethik. Stuttgart: Reclam 2016.

Frankena, William K. Analytische Ethik. München: dtv, 1972.

Gesang, Bernward. Klimaethik. Berlin: Suhrkamp, 2011.

Günzler, Carl. „Ehrfurcht vor dem Leben - Albert Schweitzers Ethik als Grundimpuls für die Umwelterziehung". In Carl Günzler, Ludwig Kerstiens, Lutz Mauermann, Franz Pöggeler, Hans-Joachim Werner, Ethik und Erziehung, 171-199. Stuttgart-Berlin-KölnMainz: Kohlhammer, 1988.

Günzler, Carl. „Ehrfurchtsethik und Umwelterziehung. Zur pädagogischen Fruchtbarkeit der Schweitzerschen Ethik“. In Albert Schweitzer heute, hrsg. von Carl Günzler, Erich Gräßer, Bodo Christ, Hans H. Eggebrecht, 110-124. Tübingen: Katzmann, 1990.

Günzler, Carl. Albert Schweitzer: Einführung in sein Denken. München: Beck 1996.

Günzler, Carl, Hans Lenk. „Ethik und Weltanschauung. Zum Neuigkeitsgehalt von Albert Schweitzers ,Kulturphilosophie III“. In Carl Günzler, Erich Gräßer, Bodo Christ, Hans H. Eggebrecht (Hg.), Albert Schweitzer heute, 17-51. Tübingen: Katzmann, 1990.

Jonas, Hans. Das Prinzip Verantwortung. Frankfurt/M: Insel, 1979.

Kant, Immanuel. Grundlegung zur Metaphysik der Sitten. In Werke, TB-Akademie-Textausgabe. Bd. IV. Berlin: de Gruyter, 1968.

Kant, Immanuel. Metaphysik der Sitten. In Werke, TB-Akademie-Textausgabe. Bd. VI. Berlin: de Gruyter, 1968.1

${ }^{66}$ Vgl. Hans Lenk, Konkrete Humanität (Frankfurt: Suhrkamp, 1998); Hans Lenk, Albert Schweitzer - Ethik als konkrete Humanität (Münster: LIT, 2000). 
Pobrane z czasopisma http://kulturaiwartosci.journals.umcs.pl

Kant, Immanuel. Kritik der praktischen Vernunft. In Werke, TB-akademie-Textausgabe. Bd. VI. Berlin: de Gruyter, 1968.

Kant, Immanuel. Die Religion innerhalb der Grenzen der bloßen Vernunft. In Werke, TB-Akademie-Textausgabe. Bd. VI. Berlin: de Gruyter, 1968.

Leffingwell Albert. “An ethical basis for humanity to animals”. Arena 10 (1894).

Lenk, Hans. Pragmatische Vernunft. Stuttgart: Reclam 1979.

Lenk, Hans. „Verantwortung für die Natur“. Allgemeine Zeitschrift für Philosophie 8, H. 3 (1983): 1-18.

Lenk, Hans. Tagebuch einer Rückreise an einen nie zuvor besuchten Ort: Lambarene. Stuttgart: Radius 1990.

Lenk, Hans. Einführung in die angewandte Ethik. Stuttgart: Kohlhammer 1997.

Lenk, Hans. Konkrete Humanität. Frankfurt: Suhrkamp 1998.

Lenk, Hans. Albert Schweitzer - Ethik als konkrete Humanität. Münster: LIT 2000.

Lenk, Hans. Global TechnoScience and Responsibility. Berlin: LIT 2007.

Lenk, Hans. Umweltverträglichkeit und Menschenzuträglichkeit. Karlsruhe: KIT 2009.

Lenk, Hans. Das flexible Vielfachwesen. Weilerswist: Velbrück 2010 (2. A. 2011).

Lenk, Hans. Human-soziale Verantwortung. Bochum-Freiburg: projektverlag, 2015.

Lenk, Hans. Humanité et responsabilité. Bochum-Freiburg: projektverlag, 2016.

Lenk, Hans. Human zwischen Öko-Ethik und Ökonomik. Bochum: projektverlag, 2018.

Lenk, Hans. Not a Long Way to Concrete Humanity? Bochum-Freiburg: projektverlag 2019.

Lenk, Hans, Matthias Maring. Natur - Umwelt - Ethik. Münster: LIT, 2003.

Luy, Jörg. „Welche Rechte haben Tiere?“, Spektrum der Wiss., Dez. 2016: 80-84.

Lenk, Hans, Günter Ropohl (Hg.). Technik und Ethik. Stuttgart: Reclam, 1987 (2. A. 1989).

Martensen, Hans Lassen. Die christliche Ethik (Dän. Orig. 1871-1878), 2 Bd. Karlsruhe: 1887.

Meyer-Abich, Klaus Michael. Frieden mit der Natur. Freiburg: Herder, 1979.

Passmore, John. „Den Unrat beseitigen“. In Ökologie und Ethik, hrsg. von Dieter Birnbacher, 207-246. Stuttgart: Reclam, 1980.

Lenk, Hans. „Herausforderung der Ethik durch technologische Macht“. Bitburger Gespräche 1981, Gesellschaft für Rechtspolitik Trier. München: Beck.

Platon, Kritias. In Sämtliche Werke, Bd. 5. Hamburg: Rowohlt 1959.

Regan, Tom, Peter Singer (Hg.). Animal Rights and Human Obligations. Englewood Cliffs: Free Press, 1976.

Reiner, Hans. „Die Zukunft der Ethik Albert Schweitzers“. Journal of Value Inquiry 1968, issue 2: 157-62.

Schwantje, Magnus. Tiermord und Menschenmord. Berlin, 1919.

Schwantje, Magnus Tierschlachtung und Krieg. Berlin, 1928 (2. erw. A.u.d.T.: Ehrfurcht vor dem Leben. Brüderlichkeit und Vegetarismus. Zürich: Verlag Volksgesundheit, 1949).

Schweitzer, Albert. Gesammelte Werke. 5 Bde. München: Beck, o. J.

Schweitzer, Albert. Kultur und Ethik. 2. A. München: C.H. Beck, 1960 (Orig. 1923).

Schweitzer, Albert. Was sollen wir tun? Heidelberg: Lambert-Schneider, 1974. 
Schweitzer, Albert. Wie wir überleben können, hrsg. von Harald Schützeichel. Freiburg: Herder, 1994.

Schweitzer, Albert. Aus meinem Leben und Denken. Hamburg: F. Meiner, 1980.

Schweitzer, Albert. Die Weltanschauung der Ehrfurcht vor dem Leben. Kulturphilosophie III, hrsg. von Claus Günzler, Johann Zürcher. 2 Bde. München: Beck, 1999.

Spaemann, Robert. „Technischer Eingriff in die Natur als Problem der politischen Ethik“. In Ökologie und Ethik, hrsg. von Dieter Birnbacher, 180-206. Stuttgart: Reclam, 1980.

\section{Streszczenie}

\section{Odpowiedzialność za przyrodę, humanizm i szacunek dla życia} od Kanta do Schweitzera

Międzyludzka etyczna odpowiedzialność i obowiązki wobec innych istot żywych według Alberta Schweitzera etyki „szacunku dla życia”. Poszerzona, ogólna i praktyczna odpowiedzialność za „przyrodę” - w szczególności za ingerencję i szkody w ekosystemach w obliczu narastającego kryzysu klimatycznego i zagrożeń ekologicznych. Co ma dziś oznaczać imperatyw „konkretnego humanizmu” i jak możemy urzeczywistnić oraz, co ważniejsze, zmaterializować takie cele i wartości? Na koniec pewne praktyczne tezy normatywne.

Słowa kluczowe: ludzka odpowiedzialność, odpowiedzialność za przyrodę, szacunek dla życia, „konkretny humanizm”

\section{Summary}

Responsibility for Nature, Humanity and Reverence for Life from Kant to Schweitzer

Ethical/moral responsibility between humans and duties/obligations for other creatures after Albert Schweitzer's ethics of "Reverence for Life". Extended responsibility for "Nature" in general and practice - in particular for encroaching on and intruding in ecosystems facing the extant climate crisis and ecological dangers. What do the imperatives of "concrete humanity" or practical "humaneness" mean - and how can we realize and, more important, materialize these aims and values? Some practice-oriented theses are sketched out.

Keywords: Responsibility for humans, duties regarding natural creatures, extended responsibilities, "concrete humanity" 
Pobrane z czasopisma http://kulturaiwartosci.journals.umcs.pl

Data: 26/04/2023 11:33:15

Hans Lenk, Naturverantwortung, Humanität und Lebensehrfurcht ...

Informacje o Autorze:

HANS LENK, profesor emerytowany, Instytut Technologii w Karlsruhe, Niemcy; e-mail: hans.lenk@kit.edu

\section{(cc) EY-NG-ND}

